\title{
Deconstructing Multiantenna Fading Channels
}

\author{
Akbar M. Sayeed, Senior Member, IEEE
}

\begin{abstract}
Accurate and tractable channel modeling is critical to realizing the full potential of antenna arrays in wireless communications. Current approaches represent two extremes: idealized statistical models representing a rich scattering environment and parameterized physical models that describe realistic scattering environments via the angles and gains associated with different propagation paths. However, simple rules that capture the effects of scattering characteristics on channel capacity and diversity are difficult to infer from existing models. In this paper, we propose an intermediate virtual channel representation that captures the essence of physical modeling and provides a simple geometric interpretation of the scattering environment. The virtual representation corresponds to a fixed coordinate transformation via spatial basis functions defined by fixed virtual angles. We show that in an uncorrelated scattering environment, the elements of the channel matrix form a segment of a stationary process and that the virtual channel coefficients are approximately uncorrelated samples of the underlying spectral representation. For any scattering environment, the virtual channel matrix clearly reveals the two key factors affecting capacity: the number of parallel channels and the level of diversity. The concepts of spatial zooming and aliasing are introduced to provide a transparent interpretation of the effect of antenna spacing on channel statistics and capacity. Numerical results are presented to illustrate various aspects of the virtual framework.
\end{abstract}

Index Terms-Beamforming, capacity, channel modeling, diversity, fading, MIMO channels, scattering, spectral representation.

\section{INTRODUCTION}

A NTENNA arrays hold great promise for bandwidth-efficient communication over the harsh wireless channel. Recent studies have indicated linear increase in capacity with the number of antennas [1], [2]. Maximal exploitation of antenna arrays in wireless communication necessitates accurate yet tractable modeling of the multi-input multi-output (MIMO) channel coupling the transmitter and receiver. Existing models represent two extreme approaches. On the one hand is a widely used statistical model that is an idealized abstraction of spatial propagation characteristics and assumes independent fading between different transmit-receive element pairs. This model has been heavily used in capacity calculations (see, e.g., [1] and [2]) and in the development of space-time coding techniques (see, e.g., [3]). On the other hand are parametric physical models, inspired by array processing techniques, that explicitly model

Manuscript received June 10, 2001; revised May 29, 2002. This work was supported in part by the National Science Foundation under Grants CCR-9875805, ECS-9979408, and CCR-0113385 and by the Office of Naval Research under Grant N00014-01-1-0825. The associate editor coordinating the review of this paper and approving it for publication was Dr. Thomas L. Marzetta.

The author is with the Department of Electrical and Computer Engineering, University of Wisconsin-Madison, Madison, WI 53706 USA (e-mail akbar@engr.wisc.edu).

Publisher Item Identifier 10.1109/TSP.2002.803324 signal copies arriving from different directions (see, e.g., [4]). Although these models are more accurate descriptions of the actual propagation environment, they are nonlinear in spatial angles, thereby making it rather difficult to incorporate them in transceiver design and capacity computations. Furthermore, the two approaches to MIMO channel modeling exist in virtual isolation. A connection between them is very much desirable so that insights derived from them can be cross-leveraged.

The two main characteristics of fading spatial channels from a communication theoretic viewpoint are the capacity and $d i$ versity afforded by the scattering environment. Physical models relate the scattering environment to the channel coefficients and dictate their statistics. The statistics, in turn, determine the diversity and capacity afforded by the channel. There have been some recent attempts at bridging the gap between the two modeling philosophies (see, e.g., [5]-[7]). However, simple rules that capture the effects of scattering and array characteristics on capacity and diversity are difficult to draw in general. Some qualitative trends, such as the decorrelation of channel coefficients with increased antenna spacing, can be inferred and have been observed in practice.

In this paper, we propose a new intermediate virtual channel representation that keeps the essence of physical modeling without its complexity, provides a tractable linear channel characterization, and offers a simple and transparent interpretation of the effects of scattering and array characteristics on channel capacity and diversity. The virtual representation is analogous to representing the channel in beamspace or the wavenumber domain, which are concepts that have been widely studied in array processing [8]. Specifically, the virtual representation describes the channel with respect to fixed spatial basis functions defined by fixed virtual angles that are determined by the spatial resolution of the arrays. Consider a $Q \times P$ matrix with elements $\{H(m, n)\}$ representing a channel with $P$ transmit and $Q$ receive antennas. As we will see, for uniformly spaced virtual angles, $\{H(m, n)\}$ are related to the virtual channel coefficients $\left\{H_{V}(q, p)\right\}$ via a two-dimensional (2-D) Fourier transform

$$
\begin{aligned}
H(m, n)= & \frac{1}{\sqrt{P Q}} \sum_{q=-\tilde{Q}}^{\tilde{Q}} \sum_{p=-\tilde{P}}^{\tilde{P}} H_{V}(q, p) \\
& \times e^{-j 2 \pi q m / Q} e^{j 2 \pi p m / P} \\
0 \leq & \leq Q-1, \quad 0 \leq n \leq P-1
\end{aligned}
$$

where $\tilde{Q}=(Q-1) / 2$ and $\tilde{P}=(P-1) / 2$. This paper studies the structure and statistics of $\left\{H_{V}(q, p)\right\}$ imposed by physical scattering characteristics.

The deceptively simple Fourier relation (1) yields many useful insights. First, in an uncorrelated scattering environment, $\{H(m, n)\}$ constitute a segment of a wide sense 
stationary process, and $\left\{H_{V}(q, p)\right\}$ are samples of the underlying spectral representation (and, hence, are approximately uncorrelated). Second, the virtual representation yields an insightful "imaging" interpretation of the scattering geometry; a realistic channel consisting of a superposition of scattering clusters with limited angular spreads is represented by the virtual matrix $\boldsymbol{H}_{V}$ consisting of nonvanishing submatrices corresponding to different clusters. Combined with the uncorrelated nature of $\left\{H_{V}(q, p)\right\}$, this yields a simple and intuitive relationship between an arbitrary scattering environment and the corresponding channel statistics, capacity, and diversity. In particular, the structure of $\boldsymbol{H}_{V}$ provides a simple interpretation of the effect of scattering on the two key factors affecting capacity: the number of parallel channels and the level of diversity associated with each parallel channel. Third, the virtual representation reveals that all scatterers are not created equal! It induces a virtual partitioning of scattering paths that explicitly exposes their contribution to capacity and diversity. Finally, via the concepts of spatial zooming and aliasing, the virtual framework also provides a transparent picture of the effect of antenna spacing on channel statistics and capacity.

Section II presents a general physical model for spatial MIMO channels, introduces the virtual channel representation, and develops the relationship between the two. Section III discusses the imaging interpretation of the virtual channel matrix in clustered scattering environments. Section IV characterizes the statistics of the virtual channel matrix imposed by a given scattering environment. In Section V, we use the virtual representation to characterize the effects of antenna spacing on capacity for any given scattering environment. Section VI discusses capacity calculations via the virtual representation and includes numerical examples to illustrate various aspects of the framework. Section VII contains some concluding remarks and directions for future work.

\section{Multiantenna Channel Modeling}

Consider a transmitter array with $P$ elements and a receiver array with $Q$ elements. In the absence of noise, the transmitted and received signals are related as

$$
\boldsymbol{x}=\boldsymbol{H s}
$$

where

$s \quad P$-dimensional transmitted signal;

$\boldsymbol{x} \quad Q$-dimensional received signal;

$\boldsymbol{H}$ channel matrix coupling the transmitter and receiver elements.

We index entries of $\boldsymbol{H}$ as $H(m, n): m=0,1, \ldots, Q-1$, $n=0,1, \ldots, P-1$. Most capacity calculations assume that $\boldsymbol{H}$ consists of independent, identically distributed (iid) Gaussian random variables, which is an idealized, rich scattering environment (see, e.g., [1], [2]). Our objective is to impose structure on $\boldsymbol{H}$ by modeling the scattering characteristics of physical channels. This would in turn enable us to assess channel capacity in realistic scattering environments and to study the effect of antenna spacing and scattering characteristics on capacity and diversity. Our workhorse for attaining this objective is the virtual channel representation introduced in Section II-C

\section{A. Uniform Linear Antenna Arrays}

In this paper, for simplicity of exposition we focus on one-dimensional (1-D) uniform linear arrays (ULAs) of antennas at both the transmitter and receiver and consider far-field scattering characteristics, that is, the scatterers are sufficiently far from both the transmitter and receiver. The essential ideas apply to arbitrary array geometries as well. Let $d_{T}$ and $d_{R}$ denote the antenna spacing at the transmitter and receiver, respectively. Then, the channel matrix can be described via the array steering and response vectors given by

$$
\begin{aligned}
& \boldsymbol{a}_{T}\left(\theta_{T}\right)=\frac{1}{\sqrt{P}}\left[1, e^{-j 2 \pi \theta_{T}}, \ldots, e^{-j 2 \pi(P-1) \theta_{T}}\right]^{T} \\
& \boldsymbol{a}_{R}\left(\theta_{R}\right)=\frac{1}{\sqrt{Q}}\left[1, e^{-j 2 \pi \theta_{R}}, \ldots, e^{-j 2 \pi(Q-1) \theta_{R}}\right]^{T}
\end{aligned}
$$

where $\theta$ and $\phi$ are related as

$$
\theta=\frac{d \sin (\phi)}{\lambda}=\alpha \sin (\phi), \quad \phi=\sin ^{-1}\left(\frac{\theta}{\alpha}\right)
$$

$\lambda$ is the wavelength of propagation, and $\alpha=d / \lambda$ is the normalized antenna spacing. The angle $\phi$ is measured relative to the horizontal axis (see Figs. 2 and 3). The vector $\boldsymbol{a}_{R}\left(\theta_{R}\right)$ represents the signal response at the receiver array due to a point source in the direction $\theta_{R}$. Similarly, $\boldsymbol{a}_{T}\left(\theta_{T}\right)$ represents the array weights needed to transmit a beam focussed in the direction $\theta_{T}$. We note that due to the finite array aperture, the receiver array collects some signals from directions in the neighborhood of $\theta_{R}$, and the transmit array couples energy at angles in the neighborhood of $\theta_{T}$ as well.

Periodicity in $\theta$ : Note that (4) defines a one-to-one map between $-\pi / 2 \leq \phi<\pi / 2$ and $-\alpha \leq \theta<\alpha$. However, the steering and response vectors in (3) are periodic in $\theta$ with period 1 . Throughout the paper, we will consider the principal period $[-0.5,0.5)$ for $\theta$. This implies that for $\alpha>0.5$, scatterers outside the range $\phi \in\left[-\sin ^{-1}(0.5 / \alpha), \sin ^{-1}(0.5 / \alpha)\right)$ alias into the principle period for $\theta$. This observation, which is illustrated in Fig. 1, is fundamental to understanding the effect of antenna spacing on capacity, as discussed in Section V. We will develop our channel representation framework in the spatial variable $\theta$.

\section{B. Physical Modeling of Scattering Environment}

For ULAs at the transmitter and receiver, the channel matrix $H$ can be generally modeled as

$$
\begin{aligned}
\boldsymbol{H}= & \int_{-\alpha_{R}}^{\alpha_{R}} \int_{-\alpha_{T}}^{\alpha_{T}} G\left(\theta_{R}, \theta_{T}\right) \\
& \times \boldsymbol{a}_{R}\left(\theta_{R}\right) \boldsymbol{a}_{T}^{H}\left(\theta_{T}\right) d \theta_{R} d \theta_{T} \\
H(m, n)= & \frac{1}{\sqrt{P Q}} \int_{-\alpha_{R}}^{\alpha_{R}} \int_{-\alpha_{T}}^{\alpha_{T}} G\left(\theta_{R}, \theta_{T}\right) \\
& \times e^{-j 2 \pi \theta_{R} m} e^{j 2 \pi \theta_{T} n} d \theta_{R} d \theta_{T}
\end{aligned}
$$

where $G\left(\theta_{R}, \theta_{T}\right)$ represents the physical scattering, and we call it the spatial spreading function. However, due to the periodicity of the steering and response vectors, the range of integrals in (5) can be replaced with the principal unit period for $\theta$. More specifically, define $k_{R}=\left\lfloor\alpha_{R}+0.5\right\rfloor, k_{T}=\left\lfloor\alpha_{T}+0.5\right\rfloor$, and 


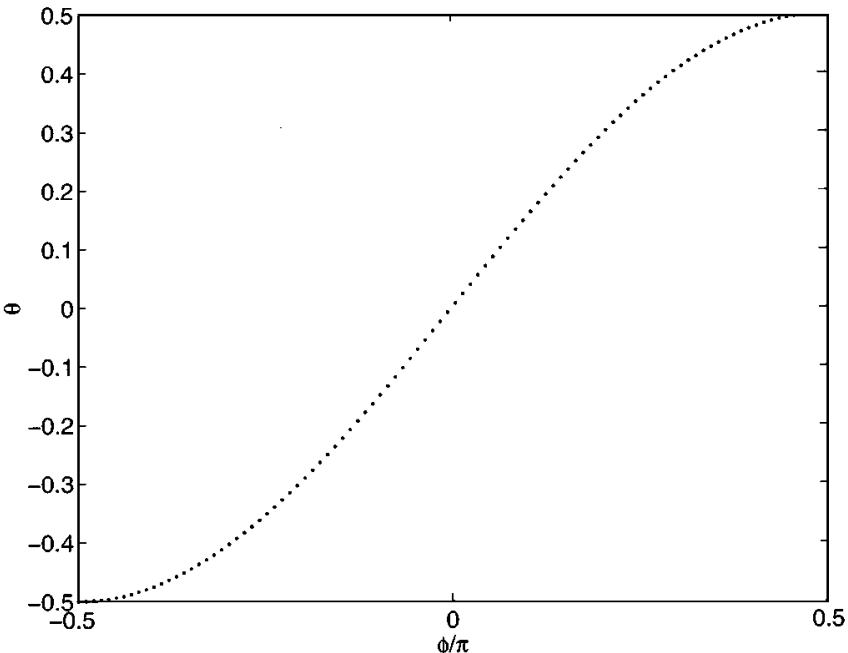

(a)

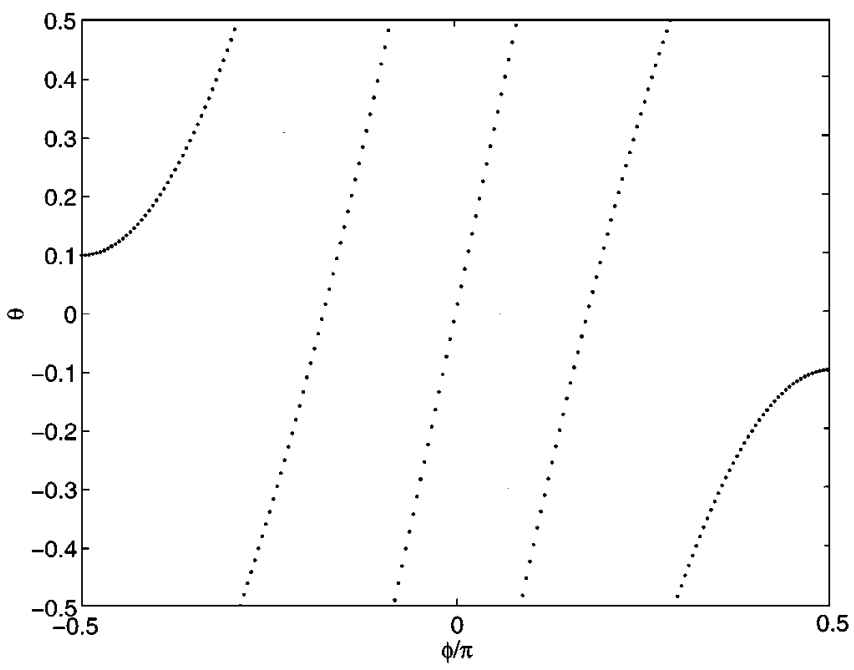

(b)

Fig. 1. Plots of $\theta$ as a function of $\phi$ as defined in (4). (a) $\alpha=0.5$. (b) $\alpha=1.9$

$G\left(\theta_{R}, \theta_{T}\right)=0$ for $\left|\theta_{R}\right|>\alpha_{R},\left|\theta_{T}\right|>\alpha_{T}$. Then, (5) can be equivalently written as

$$
\begin{aligned}
\boldsymbol{H}= & \int_{-1 / 2}^{1 / 2} \int_{-1 / 2}^{1 / 2} \hat{G}\left(\theta_{R}, \theta_{T}\right) \\
& \times \boldsymbol{a}_{R}\left(\theta_{R}\right) \boldsymbol{a}_{T}^{H}\left(\theta_{T}\right) d \theta_{R} d \theta_{T} \\
\hat{G}\left(\theta_{R}, \theta_{T}\right)= & G\left(\theta_{R} \bmod 1, \theta_{T} \bmod 1\right) \\
= & \sum_{m_{1}=-k_{R}}^{k_{R}} \sum_{m_{2}=-k_{T}}^{k_{T}} G\left(\theta_{R}-m_{1}, \theta_{T}-m_{2}\right)
\end{aligned}
$$

where $\theta \bmod 1$ denotes the value of $\theta$ in the principal period $[-0.5,0.5)$. As evident from (6) and (7), $H$ is a 2-D Fourier transform of $\hat{G}\left(\theta_{R}, \theta_{T}\right)$. We note that in many realistic environments, $G\left(\theta_{R}, \theta_{T}\right)$ is nonvanishing in smaller regions corresponding to scattering clusters with limited angular spreads, as illustrated in Fig. 2. Each cluster is represented by a nonvanishing subkernel of $G\left(\theta_{R}, \theta_{T}\right)$ with support $S_{R} \times S_{T}, S_{T} \subset$ $\left[-\alpha_{T}, \alpha_{T}\right]$, and $S_{R} \subset\left[-\alpha_{R}, \alpha_{R}\right]$. Clustered scattering environments are discussed in more detail in Section III.

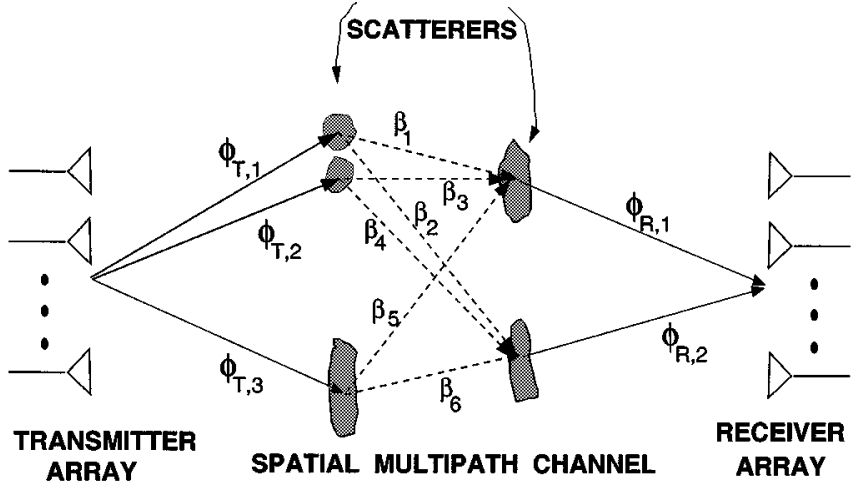

Fig. 2. Schematic illustrating physical channel modeling. Each scattering path is associated with a fading gain $\left(\beta_{l}\right)$ and a unique pair of transmit and receive angles $\left(\phi_{T, l}, \phi_{R, l}\right)$ corresponding to scatterers distributed within the angular spreads.

A widely used discrete physical model, which is a special case of (5), is illustrated in Fig. 2 and is given by

$$
\boldsymbol{H}=\sum_{l=1}^{L} \beta_{l} \boldsymbol{a}_{R}\left(\theta_{R, l}\right) \boldsymbol{a}_{T}^{H}\left(\theta_{T, l}\right)=\boldsymbol{A}_{R}\left(\underline{\theta}_{R}\right) \boldsymbol{H}_{P} A_{T}^{H}\left(\underline{\theta}_{T}\right)
$$

which corresponds to

$$
G\left(\theta_{R}, \theta_{T}\right)=\sum_{l=1}^{L} \beta_{l} \delta\left(\theta_{R}-\theta_{R, l}\right) \delta\left(\theta_{T}-\theta_{T, l}\right)
$$

in (5). In the above model, the transmitter and receiver are coupled via propagation along $L$ paths with $\left\{\theta_{T, l}\right\}$ and $\left\{\theta_{R, l}\right\}$ as the spatial angles seen by the transmitter and receiver, respectively, and $\left\{\beta_{l}\right\}$ as the corresponding independent path gains. In the matrix representation in (9), $\boldsymbol{A}_{R}\left(\underline{\theta}_{R}\right)=\left[\boldsymbol{a}_{R}\left(\theta_{R, 1}\right), \ldots, \boldsymbol{a}_{R}\left(\theta_{R, L}\right)\right](Q \times L)$,

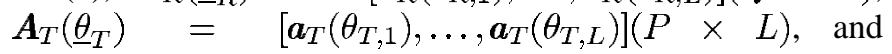
$\boldsymbol{H}_{P}=\operatorname{diag}\left(\beta_{1}, \ldots, \beta_{L}\right)$ is an $L \times L$ diagonal matrix. In the spirit of (8), the discrete model may also be expressed as

$\hat{G}\left(\theta_{R}, \theta_{T}\right)=\sum_{l=1}^{L} \beta_{l} \delta\left(\theta_{R}-\theta_{R, l} \bmod 1\right) \delta\left(\theta_{T}-\theta_{T, l} \bmod 1\right)$.

Note that the discrete model (9) is linear in the path gains $\left\{\beta_{l}\right\}$ but nonlinear in the spatial angles $\left\{\theta_{R, l}, \theta_{T, l}\right\}$.

\section{Virtual Channel Representation}

The finite dimensionality of the spatial signal space ${ }^{1}$ can be exploited to develop a linear virtual channel representation that uses spatial beams in fixed virtual directions. This is similar to beamspace and wavenumber domain in array processing literature [8]. Without loss of generality, we assume that both $P$ and $Q$ are odd and define: $\tilde{Q}=(Q-1) / 2$ and $\tilde{P}=(P-1) / 2$.

The virtual channel representation, which is illustrated in Fig. 3, can be expressed as

$$
\boldsymbol{H}=\sum_{q=-\tilde{Q}}^{\tilde{Q}} \sum_{p=-\tilde{P}}^{\tilde{P}} H_{V}(q, p) \boldsymbol{a}_{R}\left(\tilde{\theta}_{R, q}\right) \boldsymbol{a}_{T}^{H}\left(\tilde{\theta}_{T, p}\right)=\tilde{\boldsymbol{A}}_{R} \boldsymbol{H}_{V} \tilde{\boldsymbol{A}}_{T}^{H}
$$

where the matrices $\tilde{A}_{R}=\left[\boldsymbol{a}_{R}\left(\tilde{\theta}_{R,-\tilde{Q}}\right), \ldots, \boldsymbol{a}_{R}\left(\tilde{\theta}_{R, \tilde{Q}}\right)\right](Q \times Q)$ and $\tilde{A}_{T}=\left[\boldsymbol{a}_{T}\left(\tilde{\theta}_{T,-\tilde{P}}\right), \ldots, \boldsymbol{a}_{T}\left(\tilde{\theta}_{T, \tilde{P}}\right)\right](P \times P)$ are defined by

${ }^{1}$ Due to finite number of antenna elements and finite array aperture. 


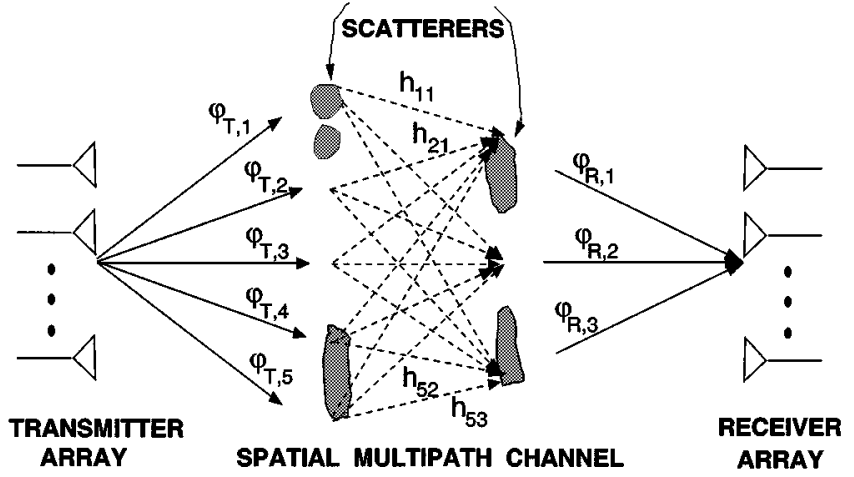

Fig. 3. Schematic illustrating virtual channel representation of the scattering environment depicted in Fig. 2. The virtual angles are fixed a priori, and their spacing defines the spatial resolution. The channel is characterized by the virtual coefficients $\left\{H_{V}(q, p)=h_{q, p}\right\}$ that couple the $P$ virtual transmit angles $\left\{\varphi_{T, p}\right\}$ with the $Q$ virtual receive angles $\left\{\varphi_{R, q}\right\}$.

the fixed virtual angles $\left\{\tilde{\theta}_{R, q}\right\}$, and $\left\{\tilde{\theta}_{T, p}\right\}$ and are full-rank. The $Q \times P$ matrix $\boldsymbol{H}_{V}$ is the virtual channel representation. In contrast to the discrete model (9), the virtual representation is linear and is characterized by $H_{V}\left(\tilde{A}_{R}\right.$ and $\tilde{A}_{T}$ are fixed). However, $H_{V}$ is not diagonal in general.

Uniform sampling of the principal $\theta$ period is a natural choice for virtual spatial angles

$$
\begin{aligned}
& \tilde{\theta}_{R, q}=\frac{q}{Q}, \quad-\tilde{Q} \leq q \leq \tilde{Q} \\
& \tilde{\theta}_{T, p}=\frac{p}{P}, \quad-\tilde{P} \leq p \leq \tilde{P}
\end{aligned}
$$

which results in the steering/response vectors (3) being sinusoids with frequencies $\tilde{\theta}_{T, p} / \tilde{\theta}_{R, q}$ and yields unitary matrices $\tilde{A}_{R}$ and $\tilde{A}_{T}$, discrete Fourier transform matrices, in fact. The corresponding fixed angles in the $\phi$ domain are

$$
\begin{aligned}
& \varphi_{T, p}=\sin ^{-1}\left(\frac{\tilde{\theta}_{T, p}}{\alpha_{T}}\right)=\sin ^{-1}\left(\frac{p}{P \alpha_{T}}\right) \\
& \varphi_{R, q}=\sin ^{-1}\left(\frac{\tilde{\theta}_{R, q}}{\alpha_{R}}\right)=\sin ^{-1}\left(\frac{q}{Q \alpha_{R}}\right)
\end{aligned}
$$

as illustrated in Fig. 4 for different values of $\alpha$. The virtual channel coefficients $\left\{H_{V}(q, p)\right\}$ represent the coupling between the $P$ virtual transmit angles $\left\{\varphi_{T, p}\right\}$ and the $Q$ virtual receive angles $\left\{\varphi_{R, q}\right\}$. Note from Fig. 3 that the virtual representation does not distinguish between scatterers that are within the spatial resolution: the scatterers corresponding to physical angles $\phi_{T, 1}$ and $\phi_{T, 2}$ in Fig. 2, for example. Furthermore, for virtual angles where there is no scattering, the corresponding coefficients are approximately zero (e.g., $h_{21}$ and $h_{52}$ in Fig. 3).

Note from Fig. 4 that for $\alpha>0.5$, a limited spatial horizon is covered by the fixed angles $\varphi$ in (14). However, as discussed earlier and illustrated in Fig. 1, values of $\phi$ outside the limited range alias into the principal period of $\theta$.

\section{Relationship Between the Virtual Representation and Physical Models}

Since $\tilde{A}_{R}$ and $\tilde{A}_{T}$ are unitary, $\boldsymbol{H}_{V}$ is related to $\boldsymbol{H}$ as

$$
\boldsymbol{H}_{V}=\tilde{A}_{R}^{H} \boldsymbol{H} \tilde{A}_{T}
$$

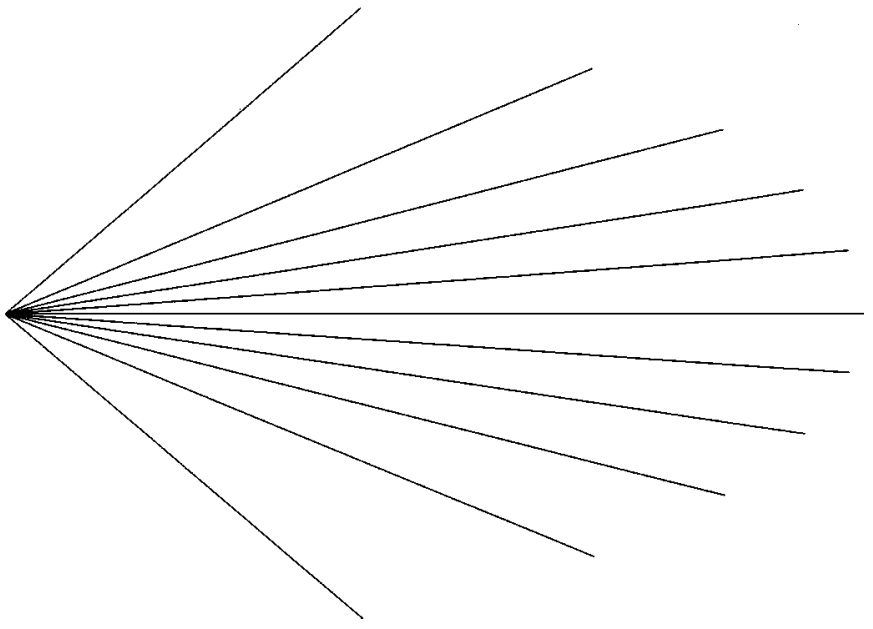

(a)

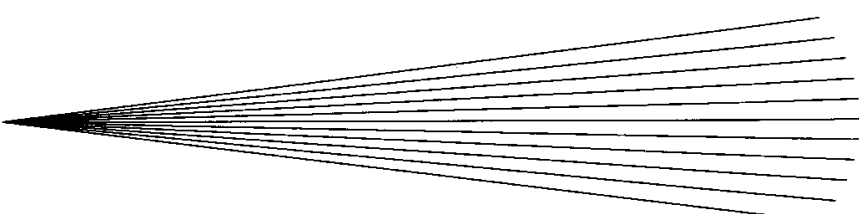

(b)

Fig. 4. Schematic illustrating beams in the $\phi$ domain corresponding to the fixed virtual angles for different values of $\alpha$. The plots correspond to an 11-element ULA. (a) $\alpha=0.5$. (b) $\alpha=1.5$.

and thus, $\boldsymbol{H}_{V}$ is unitarily equivalent to $\boldsymbol{H}$ and captures all channel information. In fact, $\boldsymbol{H}_{V}$ is a 2-D discrete Fourier transform of $\boldsymbol{H}$ [see (1))]. From (15), the elements of $\boldsymbol{H}_{V}$ are related to the physical model (7) as

$$
\begin{aligned}
H_{V}(q, p)= & \boldsymbol{a}_{R}^{H}\left(\tilde{\theta}_{R, q}\right) \boldsymbol{H} \boldsymbol{a}_{T}\left(\tilde{\theta}_{T, p}\right) \\
= & \int_{-1 / 2}^{1 / 2} \int_{-1 / 2}^{1 / 2} \hat{G}\left(\theta_{R}, \theta_{T}\right) f_{Q}\left(\theta_{R}-\frac{q}{Q}\right) \\
& \times f_{P}^{*}\left(\theta_{T}-\frac{p}{P}\right) d \theta_{R} d \theta_{T} \\
f_{Q}(\theta)= & \boldsymbol{a}_{R}^{H}(0) \boldsymbol{a}_{R}(\theta)=\frac{1}{Q} \sum_{l=0}^{Q-1} e^{-j 2 \pi \theta l} \\
= & \frac{1}{Q} e^{-j 2 \pi \theta \tilde{Q}} \frac{\sin (\pi Q \theta)}{\sin (\pi \theta)} .
\end{aligned}
$$

We note from (16) that $\left\{H_{V}(q, p)\right\}$ are samples of a smoothed version of $\hat{G}\left(\theta_{R}, \theta_{T}\right)$ at the virtual angles; the smoothing is done by the kernel $f_{Q}\left(\theta_{R}\right) f_{P}^{*}\left(\theta_{T}\right)$ that integrates to $1 / P Q$. The smoothing kernel gets peaky around the origin with increasing $P$ and $Q$. Thus, we have the following sampling approximation for sufficiently large $P$ and $Q$ :

$$
H_{V}(q, p) \approx \frac{\hat{G}\left(\frac{q}{Q}, \frac{p}{P}\right)}{P Q} .
$$

$\boldsymbol{H}_{V}$ can be expressed in matrix form for the continuous physical model as

$$
H_{V}=\int_{-1 / 2}^{1 / 2} \int_{-1 / 2}^{1 / 2} \hat{G}\left(\theta_{R}, \theta_{T}\right) \hat{\boldsymbol{a}}_{R}\left(\theta_{R}\right) \hat{\boldsymbol{a}}_{T}^{H}\left(\theta_{T}\right) d \theta_{R} d \theta_{T}
$$




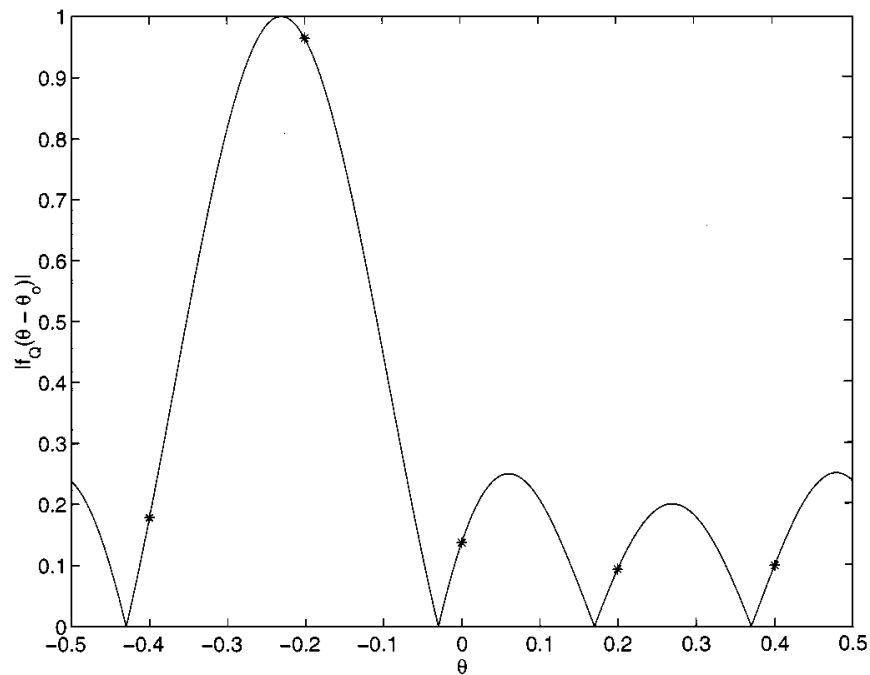

(a)

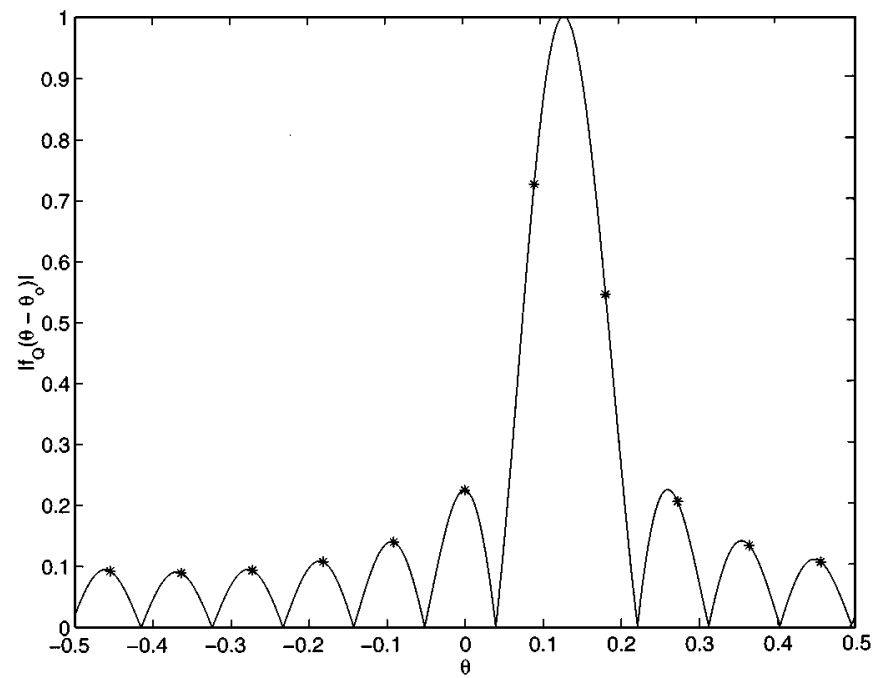

(b)

Fig. 5. Plots of $\left|f_{Q}\left(\theta-\theta_{o}\right)\right|$ versus $\theta$. $\theta_{o}$ represents the physical angle of a scatterer. Values of $\left|f_{Q}\left(q / Q-\theta_{o}\right)\right|$ corresponding to samples at the virtual angles are also marked. (a) $Q=5$, and $\theta_{o}=-0.23$. (b) $Q=11$, and $\theta_{o}=$ 0.13 .

where $\hat{\boldsymbol{a}}_{R}\left(\theta_{R}\right)=\tilde{\boldsymbol{A}}_{R}^{H} \boldsymbol{a}_{R}\left(\theta_{R}\right)$ and $\hat{\boldsymbol{a}}_{T}\left(\theta_{T}\right)=\tilde{\boldsymbol{A}}_{T}^{H} \boldsymbol{a}_{T}\left(\theta_{T}\right)$ are the projections of $a_{R}\left(\theta_{R}\right)$ and $a_{T}\left(\theta_{T}\right)$ onto the fixed virtual response and steering vectors, respectively. Similarly, for the discrete model, we have

$$
\begin{aligned}
\boldsymbol{H}_{V} & =\hat{\boldsymbol{A}}_{R}\left(\underline{\theta}_{R}\right) \boldsymbol{H}_{P} \hat{\boldsymbol{A}}_{T}^{H}\left(\underline{\theta}_{T}\right) \\
H_{V}(q, p) & =\sum_{l=1}^{L} \beta_{l} f_{Q}\left(\theta_{R, l}-\frac{q}{Q}\right) f_{P}^{*}\left(\theta_{T, l}-\frac{p}{P}\right)
\end{aligned}
$$

where $\hat{\boldsymbol{A}}_{R}\left(\underline{\theta}_{R}\right)=\tilde{\boldsymbol{A}}_{R}^{H} A_{R}\left(\underline{\theta}_{R}\right)=\left[\hat{\boldsymbol{a}}_{R}\left(\theta_{R, 1}\right), \ldots, \hat{\boldsymbol{a}}_{R}\left(\theta_{R, L}\right)\right]$ $(Q \times L)$, and $\hat{\boldsymbol{A}}_{T}\left(\underline{\theta}_{T}\right)=\tilde{A}_{T}^{H} A_{T}\left(\underline{\theta}_{T}\right)=\left[\hat{\boldsymbol{a}}_{T}\left(\theta_{T, 1}\right)\right.$, $\left.\ldots, \hat{\boldsymbol{a}}_{T}\left(\theta_{T, L}\right)\right](P \times L)$. Plots of $\left|f_{Q}\left(\theta-\theta_{o}\right)\right|$ as a function of $\theta$ are shown in Fig. 5 for two different values of $Q$ and $\theta_{o}$ ( $\theta_{\circ}$ represents the location of a physical scatterer). The values of $\left|f_{Q}\left(q / Q-\theta_{0}\right)\right|$ corresponding to samples at the virtual angles (equivalently, elements of $\left|\hat{\boldsymbol{a}}\left(\theta_{o}\right)\right|$ ) are also marked. As is evident, the projections onto virtual angles peak in the neighborhood of the physical angle $\theta_{0}$ and the peak is more pronounced for larger $Q$.

\section{E. Virtual Path Partitioning}

We now derive an approximate relationship of $\left\{H_{V}(q, p)\right\}$ to the discrete physical model via a virtual partitioning of the propagation paths. Define the following partition of path indices

$$
\begin{aligned}
S_{R, q} & =\left\{l:-\frac{1}{2 Q} \leq \theta_{R, l} \bmod 1-\frac{q}{Q}<\frac{1}{2 Q}\right\} \\
-\tilde{Q} & \leq q \leq \tilde{Q} \\
S_{T, p} & =\left\{l:-\frac{1}{2 P} \leq \theta_{T, l} \bmod 1-\frac{p}{P}<\frac{1}{2 P}\right\} \\
-\tilde{P} & \leq p \leq \tilde{P} .
\end{aligned}
$$

$S_{R, q}$ is the set of all paths whose receive angles $\theta_{R, l}$ (after shifting them into the principle $\theta$ range) are within $1 / 2 Q$ of the $q$ th virtual receive angle $\tilde{\theta}_{R, q}=q / Q . S_{T, p}$ is similarly defined with respect to virtual transmit angles. Note that

$$
\bigcup_{q} S_{R, q}=\bigcup_{p} S_{T, p}=\bigcup_{p, q}\left[S_{R, q} \cap S_{T, p}\right]=\{1,2, \ldots, L\} .
$$

Using this partition, we can express the aliased spreading function for the discrete model (11) as

$$
\begin{aligned}
\hat{G}\left(\theta_{R}, \theta_{T}\right) \approx \sum_{q=-\tilde{Q}}^{\tilde{Q}} \sum_{p=-\tilde{P}}^{\tilde{P}} & {\left[\sum_{l \in S_{R, q} \cap S_{T, p}} \beta_{l}\right] } \\
& \times \delta\left(\theta_{R}-\frac{q}{Q}\right) \delta\left(\theta_{T}-\frac{p}{P}\right)
\end{aligned}
$$

and the channel matrix via (7) as

$$
\boldsymbol{H} \approx \sum_{q=-\tilde{Q}}^{\tilde{Q}} \sum_{p=-\tilde{P}}^{\tilde{P}}\left[\sum_{l \in S_{R, q} \cap S_{T, p}} \beta_{l}\right] \boldsymbol{a}_{R}\left(\frac{q}{Q}\right) \boldsymbol{a}_{T}^{H}\left(\frac{p}{P}\right)
$$

and (21) reduces to

$$
H_{V}(q, p) \approx\left[\sum_{l \in S_{R, q} \cap S_{T, p}} \beta_{l}\right]
$$

since $f_{Q}\left(\theta_{R}-q / Q\right)$ and $f_{P}\left(\theta_{T}-p / P\right)$ are peaky around $\theta_{R}=$ $q / Q, \theta_{T}=p / P$, and $f_{Q}(0)=f_{P}(0)=1$. Equations (24) and (26) state that the scattering contribution to the virtual angle pair $\left(\tilde{\theta}_{R, q}, \tilde{\theta}_{T, p}\right)=(q / Q, p / P)$ is proportional to the number of paths whose angles $\left(\theta_{R, l}, \theta_{T, l}\right)$ lie in the rectangular virtual spatial bin of size $1 / Q \times 1 / P$ centered on $(q / Q, p / P)$ :

$$
\begin{aligned}
B_{q, p} & =\left\{\left(\theta_{R}, \theta_{T}\right):-\frac{1}{2 Q} \leq \theta_{R}-\frac{q}{Q}<\frac{1}{2 Q},\right. \\
-\frac{1}{2 P} & \left.\leq \theta_{T}-\frac{p}{P}<\frac{1}{2 P}\right\} .
\end{aligned}
$$

Based on the above path partitioning, we define the notion of distinct paths that is insightful in the contribution of paths to capacity and diversity (see Section VI). We say that two paths are distinct if they can be distinguished in either transmit or receive virtual angles, that is, they belong to distint sets $S_{R, q}$ or $S_{T, p}$. We say that two paths are strictly distinct if they can be distinguished in both transmit and receive virtual angles, that is, 
they belong to distinct sets $S_{R, q}$ and $S_{T, p}$, or, equivalently, their transmit/receive angles lie in distinct spatial bins $B_{q, p}$. Note that the number of paths that lie in each spatial bin $B_{q, p}$ affects the statistics of $H_{V}(q, p)$. Sufficiently many paths in each bin would result in Gaussian statistics via the central limit theorem. Conversely, for fixed number of paths, we would expect the statistics to deviate from Gaussian as we increase the number of antennas (and, hence, the array aperture) while keeping the spacing fixed. Fewer paths would contribute to each $B_{q, p}$ [and $H_{V}(q, p)$ ] due to the increased spatial resolution.

\section{F. Relationship to Other Models}

The physical model (5) can represent any linear channel and can account for many particular scenarios that have been studied by other researchers. For example, effects of mutual coupling can be incorporated by multiplying $H$ with a coupling matrix [9], [10]. This can be readily captured by the physical model (5) by including an appropriate coupling spreading function with $G\left(\theta_{R}, \theta_{T}\right)$ in a multiplicative fashion or by imposing appropriate statistics on $G\left(\theta_{R}, \theta_{T}\right)$. Similarly, several recent studies have used the ring model for scatterers [11] around a mobile handset to analyze the channel between an elevated base station and a mobile (see, e.g., [5], [6], [12], [13]). This can be readily accounted for (for the downlink, for example) by choosing $Q=$ 1 (mobile receiver), appropriately choosing the transmit angular spread $S_{T}$ seen by the base station, and imposing appropriate statistics on $G\left(\theta_{R}, \theta_{T}\right)$. A related effect (the so-called pinhole effect [5]) can also be accounted for by using a cascade of channels of the form (5) with appropriately chosen angular spreads and statistics.

\section{VIRTUAL REPRESENTATION OF REALISTIC CHANNELS}

Realistic scattering environments can be modeled via a superposition of clusters with limited angular spreads (see, e.g., [13]). The virtual matrix $\boldsymbol{H}_{V}$ provides an intuitively appealing representation for such environments: Different clusters correspond to different nonvanishing sub-matrices of $\boldsymbol{H}_{V}$. Equations (18) and (26) form the basis of this "imaging" interpretation. Furthermore, as we show in Section IV, the nonvanishing elements of $\boldsymbol{H}_{V}$ are approximately uncorrelated under the assumption of uncorrelated scattering.

Fig. 6 illustrates the imaging interpretation of $\boldsymbol{H}_{V}$ via contour plots of $\left|H_{V}(q, p)\right|$ for different scattering environments. $P=Q=21, \alpha_{T}=\alpha_{R}=0.5$, and $\boldsymbol{H}$ is generated via the discrete model using $L=200$ and iid zero-mean Gaussian path gains. For example, Fig. 6(a) depicts two point scatterers, and Fig. 6(b) depicts two $\pi / 8 \times \pi / 8$-wide clusters. The size of a particular sub-matrix of $\boldsymbol{H}_{V}$ is determined by the size of the corresponding cluster and the antenna spacing. To illustrate this, consider a single cluster with angular spreads $S_{R}=\left[S_{R-}, S_{R+}\right] \subset[-\pi / 2, \pi / 2)$ and $S_{T}=\left[S_{T-}, S_{T+}\right] \subset[-\pi / 2, \pi / 2)$ in the $\phi$ domain. The corresponding sub-matrix is nonvanishing for $q=Q_{-}, \ldots, Q_{+}$, $p=P_{-}, \ldots, P_{+}$, where $Q_{-} \approx\left\lfloor\alpha_{R} Q \sin \left(S_{R-}\right)\right\rfloor, Q_{+} \approx$ $\left\lceil\alpha_{R} Q \sin \left(S_{R+}\right)\right\rceil$, and similarly, $P_{-} \approx\left\lfloor\alpha_{T} P \sin \left(S_{T-}\right)\right\rfloor$ and
$P_{+} \approx\left\lceil\alpha_{T} P \sin \left(S_{T+}\right)\right\rceil .{ }^{2}$ Note that for a given spread in the $\phi$ domain, the size of the sub-matrix is larger for larger antenna spacings. The rank of the sub-matrix is given by $r \approx \min \left(Q_{+}-Q_{-}+1, P_{+}-P_{-}+1\right)$. For example, for the top left cluster in Fig. 6(b), $S_{R}=[\pi / 16,3 \pi / 16]$ and $S_{T}=[-3 \pi / 16,-\pi / 16]$. For $P=Q=21$ and $\alpha_{R}=\alpha_{T}=1 / 2$, this yields $\left(Q_{-}, Q_{+}\right)=(2,6)$ and $\left(P_{-}, P_{+}\right)=(-6,-2)$, resulting in a $5 \times 5$ sub-matrix with rank 5. The rank of $\boldsymbol{H}_{V}$ (and $\boldsymbol{H}$ ) is closely approximated by the sum of the ranks of strictly distinct sub-matrices (with disjoint supports in both transmit and receive virtual angles).

The accuracy of the imaging interpretation of $\boldsymbol{H}_{V}$ is governed by both the number of antenna elements as well as their spacing. For given spacing, the accuracy improves with the number of elements because, to a first approximation, the extent of smoothing (or spatial resolution) in (16) is determined by the widths of the mainlobes of $f_{Q}\left(\theta_{R}\right) f_{P}^{*}\left(\theta_{T}\right)-\Delta \theta_{R} \times \Delta \theta_{T}=2 / Q \times 2 / P$ - which get narrower for larger $Q$ and $P$, as is evident from Fig. 5. For given $Q$ and $P$, the accuracy improves with increased $\alpha$ since the separation between two fixed scatterers increases in $\theta$ via (4). The smoothing in (16) is a fundamental consequence of limited array apertures, and the spacing of virtual angles is commensurate with the spatial resolution. ${ }^{3}$ This has important implications regarding the effect of scattering characteristics on channel statistics, capacity, and diversity, which are exemplified by our analysis based on virtual path partitioning.

Virtual channel representation clearly reveals the two key factors that affect the capacity of the spatial channel corresponding to each cluster.

- The number of parallel channels, which is equal to the rank of the sub-matrix, is determined by the number of transmit and receive virtual angles that lie within the cluster angular spreads.

- The level of diversity associated with each parallel channel is determined by the number of virtual receive angles that couple with each virtual transmit angle, and vice versa. This depends on the nature of scattering within the cluster, as will be elaborated upon in the following.

$k$-Diagonal Virtual Modeling: To illustrate how the level of diversity is determined by the nature of scattering, let $P=Q$ and $\tilde{P}=(P-1) / 2$. Consider a single cluster covering the entire spatial horizon $\left(S_{R-}=S_{T-}=-\pi / 2\right.$, and $S_{R+}=S_{T+}=$ $\pi / 2)$. On one extreme is "diagonal scattering" $\left(\boldsymbol{H}_{V}\right.$ approximately diagonal), which is illustrated in Fig. 6(c), in which each transmit virtual angle couples with only a few corresponding virtual receive angles resulting in low diversity. A corresponding physical environment consisting of a single line of scatterers is illustrated in Fig. 7(a). On the other extreme is "maximally rich scattering" (all elements of $\boldsymbol{H}_{V}$ nonzero), which is illustrated in Fig. 6(d), in which each virtual transmit angle couples with all

\footnotetext{
${ }^{2}$ The indices $Q_{-}, Q_{+}, P_{-}, P_{+}$are defined to capture all of the energy in the cluster. Depending on how the virtual beams couple with the cluster, the coefficients on the edge of the sub-matrix may contribute relatively small power.

${ }^{3}$ Even if we have high-resolution measurements of $\hat{G}\left(\theta_{R}, \theta_{T}\right)$ available, actual system performance will be governed by a smoothed version whose resolution is commensurate with the array apertures.
} 


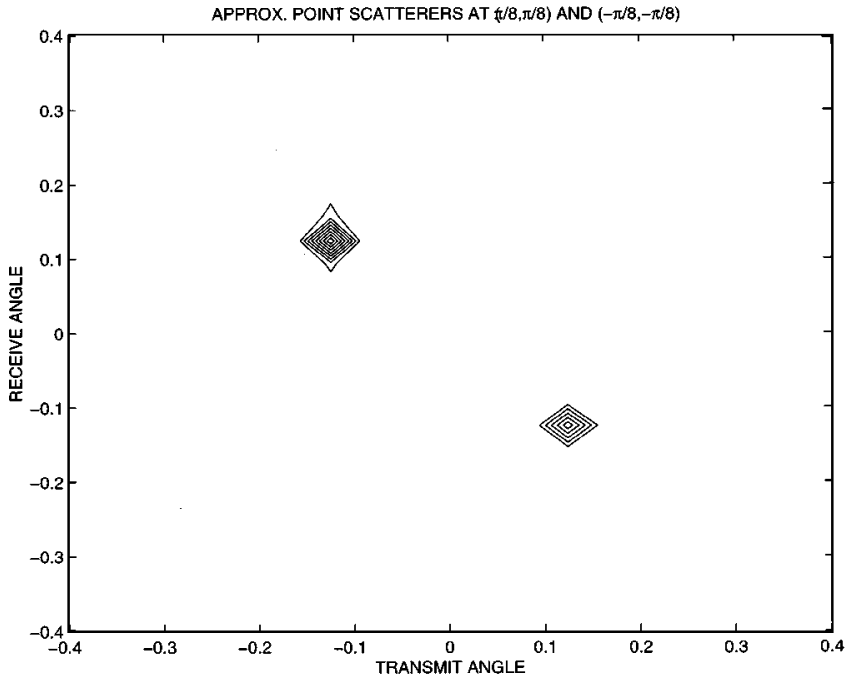

(a)

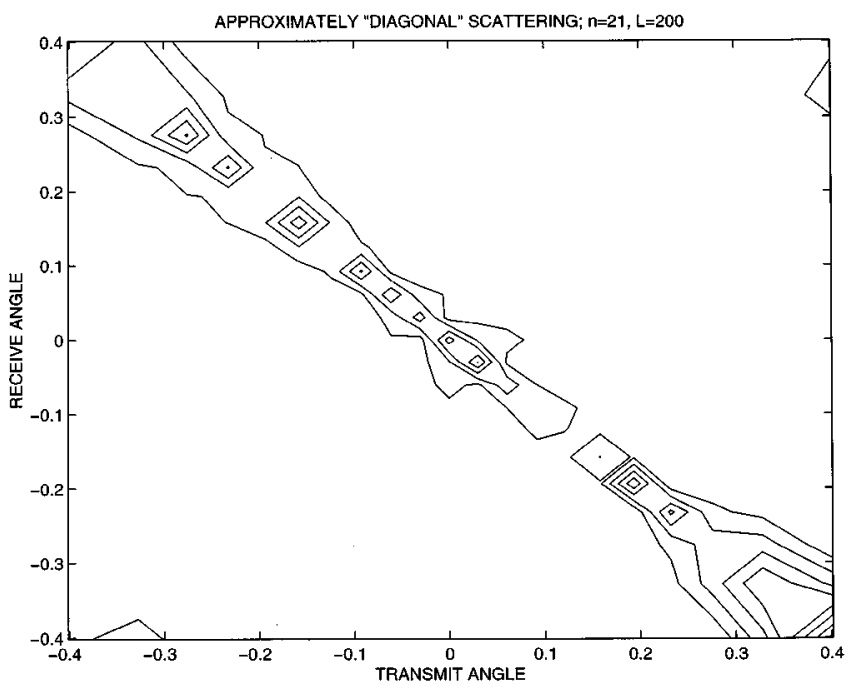

(c)

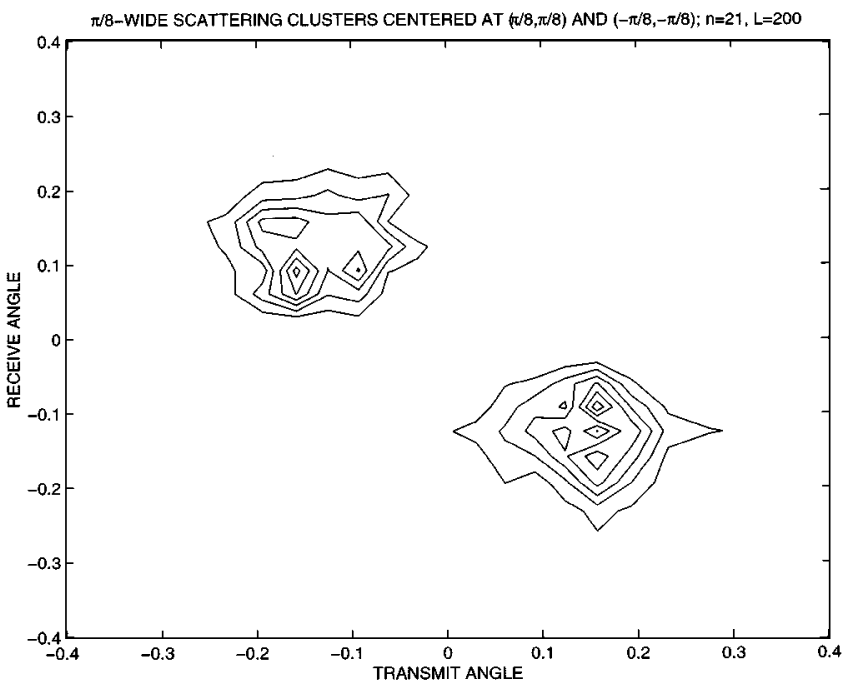

(b)

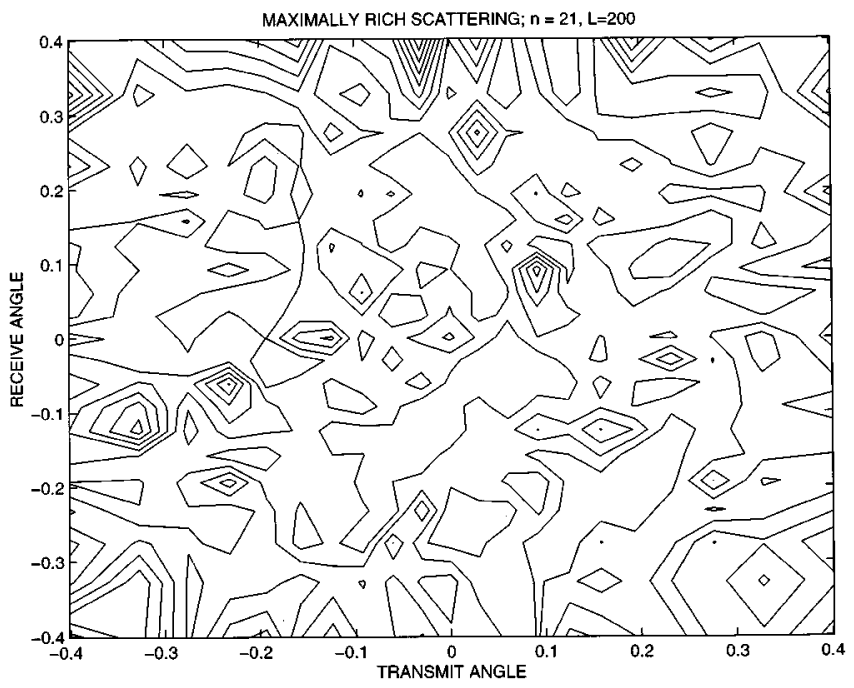

(d)

Fig. 6. "Imaging" of the scattering environment via $\boldsymbol{H}_{V} \cdot P=Q=21$ and contour plots of $\left|H_{V}(q, p)\right|$ are shown. The horizontal axis represents transmit angles (column index $p$ ), and the vertical axis represents receive angles (row index $q$ ). (a) Two point scatterers centered at $\left(\phi_{T}, \phi_{R}\right)=(\pi / 8,-\pi / 8)$ and $(-\pi / 8, \pi / 8)$. (b) Two $\pi / 8 \times \pi / 8$-wide clusters centered at $(\pi / 8,-\pi / 8)$ and $(-\pi / 8, \pi / 8)$. (c) "Diagonal" scattering, in which each transmit angle couples with only a few corresponding receive angles. (d) Maximally rich scattering, in which each transmit angle couples with all receive angles.

virtual receive angles resulting in maximum diversity. A corresponding physical geometry consisting of multiple lines of scatterers is illustrated in Fig. 7(b). This suggests that we can capture a rich class of scattering environments depicting different levels of diversity by imposing structure on the nonvanishing elements of $\boldsymbol{H}_{V}$. One possibility is the following simple $k$-diagonal virtual model that spans the two extreme cases

$$
\boldsymbol{H}_{k}=\sum_{p=-\tilde{P}}^{\tilde{P}} \sum_{q=\max (-\tilde{P}, p-k)}^{\min (\tilde{P}, p+k)} H_{V}(q, p) \boldsymbol{a}_{R}\left(\tilde{\theta}_{R, q}\right) \boldsymbol{a}_{T}^{H}\left(\tilde{\theta}_{T, p}\right)
$$

where $0 \leq k \leq P-1$ is the number of diagonals above and below the main diagonal. Diagonal scattering corresponds to $k=0$, and maximally rich scattering corresponds to $k=P-$ 1. As we elaborate in Section VI, for the same received SNR, these two channels have nearly identical ergodic capacities but radically different outage capacities due to higher diversity in the latter case. The schematic in Fig. 8 summarizes the structure of $\boldsymbol{H}_{V}$ and its implications for capacity and diversity dictated by the imaging interpretation in clustered scattering environments.

\section{Channel Statistics}

In this section, we discuss the statistics of $\boldsymbol{H}$ and $\boldsymbol{H}_{V}$ imposed by the physical model, which yields useful insights into the effect of scattering on capacity and diversity. We assume an uncorrelated Rayleigh scattering environment, that is, $\left\{G\left(\theta_{R}, \theta_{T}\right)\right\}$ is a family of zero-mean Gaussian random variables

$$
\begin{aligned}
E\left[G\left(\theta_{R}, \theta_{T}\right) G^{*}\right. & \left.\left(\theta_{R}^{\prime}, \theta_{T}^{\prime}\right)\right] \\
& =M\left(\theta_{R}, \theta_{T}\right) \delta\left(\theta_{R}-\theta_{R}^{\prime}\right) \delta\left(\theta_{T}-\theta_{T}^{\prime}\right)
\end{aligned}
$$

for some $M\left(\theta_{R}, \theta_{T}\right) \geq 0$ reflecting the channel power as a function of $\left(\theta_{R}, \theta_{T}\right)$. In line with the terminology used for temporal 


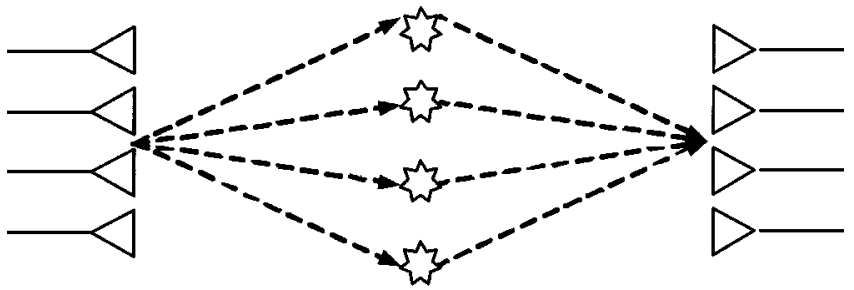

(a)

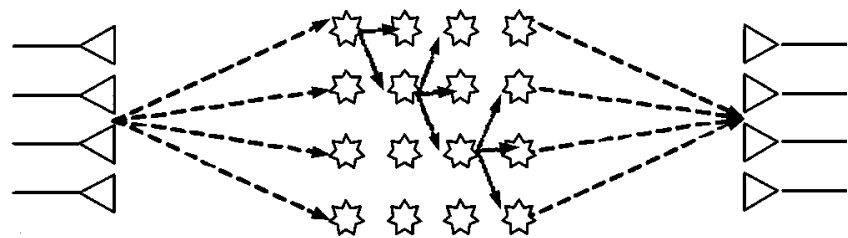

(b)

Fig. 7. Conceptual schematic illustrating physical geometry associated with "diagonal" and "maximally rich" scattering. (a) Diagonal scattering consisting of a line of scatterers. (b) Rich scattering consisting of multiple lines of scatterers.

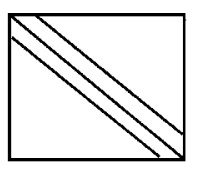

MEDIUM CAPACITY

MEDIUM DIVERSITY

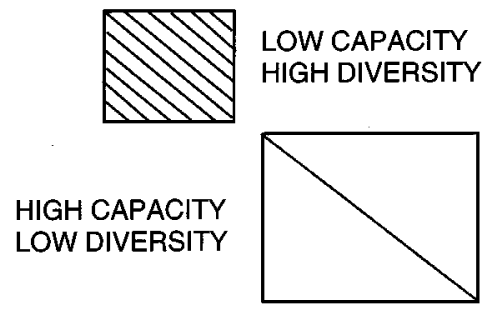

Fig. 8. Schematic depicting the decomposition of $\boldsymbol{H}_{V}$ for a clustered scattering environment into nonvanishing sub-matrices (with uncorrelated entries). Each sub-matrix is in turn modeled as a $k$-diagonal matrix, reflecting the nature of scattering in the cluster. The size of a sub-matrix determines the capacity, and the number of diagonals represents the diversity afforded by the cluster.

channels (see, e.g., [14]), we call $M\left(\theta_{R}, \theta_{T}\right)$ the spatial scattering function. It follows from (8) that for the aliased spreading function, we have

$$
\begin{aligned}
& E\left[\hat{G}\left(\theta_{R}, \theta_{T}\right) \hat{G}^{*}\left(\theta_{R}^{\prime}, \theta_{T}^{\prime}\right)\right] \\
& \quad=\hat{M}\left(\theta_{R}, \theta_{T}\right) \delta\left(\theta_{R}-\theta_{R}^{\prime}\right) \delta\left(\theta_{T}-\theta_{T}^{\prime}\right) \\
& \hat{M}\left(\theta_{R}, \theta_{T}\right)=M\left(\theta_{R} \bmod 1, \theta_{T} \bmod 1\right) \\
& \quad=\sum_{m_{1}=-k_{R}}^{k_{R}} \sum_{m_{2}=-k_{T}}^{k_{T}} M\left(\theta_{R}-m_{1}, \theta_{T}-m_{2}\right) .
\end{aligned}
$$

For the discrete physical model, the assumption of uncorrelated scattering corresponds to

$$
E\left[\beta_{l} \beta_{l^{\prime}}^{*}\right]=\sigma_{l}^{2} \delta_{l-l^{\prime}}
$$

and we have

$$
\begin{aligned}
\hat{M}\left(\theta_{R}, \theta_{T}\right)=\sum_{l=1}^{L} \sigma_{l}^{2} \delta\left(\theta_{R}-\theta_{R, l}\right. & \bmod 1) \\
& \times \delta\left(\theta_{T}-\theta_{T, l} \bmod 1\right)
\end{aligned}
$$

under the assumption that different paths correspond to distinct physical angles.

\section{A. Channel Power}

Define the total channel power as

$$
\sigma_{H}^{2}=E\left(\operatorname{trace}\left[\boldsymbol{H}^{H} \boldsymbol{H}\right]\right)=\sum_{m=0}^{Q-1} \sum_{n=0}^{P-1} E\left[|H(m, n)|^{2}\right] \text {. }
$$

In idealized statistical models (rich scattering), $E\left[|H(m, n)|^{2}\right]=1$, and $\sigma_{H}^{2}=P Q$. We now compute the power distribution in $\boldsymbol{H}$ and $\boldsymbol{H}_{V}$ imposed by the physical model.

Using (7) and (30), the channel power in (34) is given by

$$
\begin{aligned}
\sigma_{H}^{2} & =\int_{-1 / 2}^{1 / 2} \int_{-1 / 2}^{1 / 2} \hat{M}\left(\theta_{R}, \theta_{T}\right) d \theta_{R} d \theta_{T} \\
& =\sum_{l=1}^{L} \sigma_{l}^{2} \text { and } \\
E\left[|H(m, n)|^{2}\right] & =\frac{1}{P Q} \int_{-1 / 2}^{1 / 2} \int_{-1 / 2}^{1 / 2} \hat{M}\left(\theta_{R}, \theta_{T}\right) d \theta_{R} d \theta_{T} \\
& =\frac{\sigma_{H}^{2}}{P Q}
\end{aligned}
$$

where the second equality in (35) corresponds to the discrete model. We conclude that in an arbitrary scattering environment, just as in the iid case, channel power is uniformly distributed between $\{H(m, n)\}$. However, unlike the iid case, $\{H(m, n)\}$ are correlated for clustered scattering environments, as discussed in Section IV-B.

The power in $\left\{H_{V}(q, p)\right\}$ can be computed from (16) and (30) as

$$
\begin{aligned}
E\left[\left|H_{V}(q, p)\right|^{2}\right]= & \int_{-1 / 2}^{1 / 2} \int_{-1 / 2}^{1 / 2} \hat{M}\left(\theta_{R}, \theta_{T}\right)\left|f_{Q}\left(\theta_{R}-\frac{q}{Q}\right)\right|^{2} \\
& \times\left|f_{P}\left(\theta_{T}-\frac{p}{P}\right)\right|^{2} d \theta_{R} d \theta_{T} \\
\approx & \frac{\hat{M}\left(\frac{q}{Q}, \frac{p}{P}\right)}{P Q}
\end{aligned}
$$

where the last approximation is based on the fact that $\mid f_{Q}\left(\theta_{R}-\right.$ $q / Q)\left.\right|^{2}$ and $\left|f_{P}\left(\theta_{T}-p / P\right)\right|^{2}$ are peaky around $q / Q$ and $p / P$ (for sufficiently large $P, Q$ ), respectively, and integrate to $1 / Q$ and $1 / P$. Using (24) for the discrete model, it readily follows that

$$
\begin{aligned}
\hat{M}\left(\theta_{R}, \theta_{T}\right) \approx \sum_{q=-\tilde{Q} p=-\tilde{P}}^{\tilde{Q}} \sum^{\tilde{P}} & {\left[\sum_{l \in S_{R, q} \cap S_{T, p}} \sigma_{l}^{2}\right] } \\
& \times \delta\left(\theta_{R}-\frac{q}{Q}\right) \delta\left(\theta_{T}-\frac{p}{P}\right)
\end{aligned}
$$

which is an approximate partition of (33) imposed by the path partitioning in Section II-E. Thus, from (33), (37), and (39), we conclude that the power is distributed in $\left\{H_{V}(q, p)\right\}$ as

$$
\begin{aligned}
\sigma_{H}^{2} & =\sum_{q=-\tilde{Q}}^{\tilde{Q}} \sum_{p=-\tilde{P}}^{\tilde{P}} E\left[\left|H_{V}(q, p)\right|^{2}\right] \\
& \approx \sum_{q=-\tilde{Q}}^{\tilde{Q}} \sum_{p=-\tilde{P}}^{\tilde{P}} \frac{\hat{M}\left(\frac{q}{Q}, \frac{p}{P}\right)}{P Q} \\
\sigma_{H}^{2} & =\sum_{q=-\tilde{Q}} \sum_{p=-\tilde{P}}^{\tilde{Q}}\left[\sum_{l \in S_{R, q} \cap S_{T, p}} \sigma_{l}^{2}\right]
\end{aligned}
$$


where the approximation in (40) is a Riemann sum approximation to the integral in (35), (41) applies to the discrete model and, its approximation is based on virtual path partitioning. Equation (41) is consistent with (26) and states that the power in $H_{V}(q, p)$ is equal to the sum of the powers of the paths that lie in the corresponding spatial bin defined in (27).

\section{B. Channel Correlation-Spectral Representation Interpretation for $\boldsymbol{H}_{V}$}

In this section, we investigate the correlation imposed by the physical model on $\boldsymbol{H}$ and $\boldsymbol{H}_{V}$. We first consider the correlation of $\boldsymbol{H}$. From (7) and (30), we have

$$
\begin{aligned}
R_{H}( & \left.m-m^{\prime}, n-n^{\prime}\right) \\
= & E\left[H(m, n) H^{*}\left(m^{\prime}, n^{\prime}\right)\right] \\
= & \frac{1}{P Q} \int_{-1 / 2}^{1 / 2} \int_{-1 / 2}^{1 / 2} \hat{M}\left(\theta_{R}, \theta_{T}\right) \\
& \times e^{-j 2 \pi \theta_{R}\left(m-m^{\prime}\right)} \\
& \times e^{j 2 \pi \theta_{T}\left(n-n^{\prime}\right)} d \theta_{R} d \theta_{T} \\
= & \frac{1}{P Q} \sum_{l=1}^{L} \sigma_{l}^{2} e^{-j 2 \pi \theta_{R, l}\left(m-m^{\prime}\right)} \\
& \times e^{j 2 \pi \theta_{T, l}\left(n-n^{\prime}\right)} \\
\approx & \frac{1}{P Q} \sum_{q=-\tilde{Q}}^{\tilde{Q}} \sum_{p=-\tilde{P}}^{\tilde{P}}\left[\sum_{l \in S_{R, q} \cap S_{T, p}} \sigma_{l}^{2}\right] \\
& \times e^{-j 2 \pi q\left(m-m^{\prime}\right) / Q} e^{j 2 \pi p\left(n-n^{\prime}\right) / P}
\end{aligned}
$$

where (43) holds for the discrete model, and the last approximation is based on virtual path partitioning. The above expressions shows that $\{H(m, n)\}$ constitutes a segment of a 2-D widesense stationary (WSS) process since their correlation function only depends on $\left(m-m^{\prime}, n-n^{\prime}\right)$. We also conclude from (42) that $\hat{M}\left(\theta_{R}, \theta_{T}\right)$ is the power spectral density (PSD) of $R_{H}(\Delta m, \Delta n)$, and it corresponds to a line spectrum in the discrete model with different lines corresponding to angles of different paths in (43). The virtual path partitioning approximates the line spectrum in (43) with uniformly spaced lines corresponding to virtual angles in (44).

The spectral representation interpretation implies that iid $\{H(m, n)\}$ (analogous to white noise) correspond to uniform power distribution in $\hat{G}\left(\theta_{R}, \theta_{T}\right)$ over the entire range of $\left(\theta_{R}, \theta_{T}\right)$

$$
\begin{aligned}
R_{H}\left(m-m^{\prime}, n-n^{\prime}\right) & =R_{H}(0,0) \delta_{m-m^{\prime}} \delta_{n-n^{\prime}} \\
& \Longleftrightarrow \hat{M}\left(\theta_{R}, \theta_{T}\right)=\sigma_{H}^{2}, \\
-\frac{1}{2} & \leq \theta_{R}, \theta_{T}<\frac{1}{2}
\end{aligned}
$$

where $R_{H}(0,0)=\sigma_{H}^{2} / P Q$. We can also conclude from (44) that at least $L=P Q$ strictly distinct paths are needed to result in iid $\{H(m, n)\}$.

Given the WSS nature of $\{H(m, n)\}$ under uncorrelated scattering and the fact that $\boldsymbol{H}$ and $\hat{G}\left(\theta_{R}, \theta_{T}\right)$ are related via a Fourier transform [see (7)], we can interpret $G\left(\theta_{R}, \theta_{T}\right)$ as the spectral representation of $\{H(m, n)\}$ (see, e.g., [15]). Similarly, from (1) and (18), we conclude that $\left\{H_{V}(q, p)\right\}$ are uniformly spaced samples of the spectral representation. Consequently, we expect the $\left\{H_{V}(q, p)\right\}$ to be approximately ${ }^{4}$ uncorrelated, which indeed follows from (16) and (26)

$$
\begin{aligned}
E\left[H_{V}(q, p) H_{V}^{*}\left(q^{\prime}, p^{\prime}\right)\right]= & \int_{-1 / 2}^{1 / 2} \int_{-1 / 2}^{1 / 2} \hat{M}\left(\theta_{R}, \theta_{T}\right) \\
& \times f_{Q}\left(\theta_{R}-\frac{q}{Q}\right) f_{Q}^{*}\left(\theta_{R}-\frac{q^{\prime}}{Q}\right) \\
& \times f_{P}^{*}\left(\theta_{T}-\frac{p}{P}\right) \\
& \times f_{P}\left(\theta_{T}-\frac{p^{\prime}}{P}\right) d \theta_{R} d \theta_{T} \\
\approx & \frac{\hat{M}\left(\frac{q}{Q}, \frac{p}{P}\right) \delta_{q-q^{\prime}} \delta_{p-p^{\prime}}}{P Q} \\
\approx & {\left[\sum_{l \in S_{R, q} \cap S_{T, p}} \sigma_{l}^{2}\right] \delta_{p-p^{\prime}} \delta_{q-q^{\prime}} }
\end{aligned}
$$

where the last approximation holds for the discrete model under virtual path partitioning. Finally, using (1), we can explicitly relate the statistics of $\boldsymbol{H}$ and $\boldsymbol{H}_{V}$ as

$$
\begin{aligned}
R_{H}\left(m-m^{\prime}, n-n^{\prime}\right) \approx & \frac{1}{P Q} \sum_{q=-\tilde{Q}}^{\tilde{Q}} \sum_{p=-\tilde{P}}^{\tilde{P}} E\left[\left|H_{V}(q, p)\right|^{2}\right] \\
& \times e^{-j 2 \pi q\left(m-m^{\prime}\right) / Q} e^{j 2 \pi p\left(n-n^{\prime}\right) / P}
\end{aligned}
$$

which is analogous to the relationship between the PSD and the correlation function of a WSS process.

Concatenated Vector Channel Correlation Matrix: We now develop a revealing vector representation of (48). From (12), it follows that

$$
\boldsymbol{h}=\operatorname{vec}(\boldsymbol{H})=\left[\tilde{A}_{T}^{*} \otimes \tilde{A}_{R}\right] \operatorname{vec}\left(\boldsymbol{H}_{V}\right)=\left[\tilde{\boldsymbol{A}}_{T}^{*} \otimes \tilde{\boldsymbol{A}}_{R}\right] \boldsymbol{h}_{V}
$$

where vec $(H)$ represents a $Q P \times 1$ vector obtained by stacking the columns of $H$, and $\otimes$ represents the Kronecker product [16]. Here, we have used the identity $\operatorname{vec}(\boldsymbol{A D B})=\left[\boldsymbol{B}^{T} \otimes \boldsymbol{A}\right] \operatorname{vec}(\boldsymbol{D})$ [16]. Let $\boldsymbol{R}=E\left[\boldsymbol{h} \boldsymbol{h}^{H}\right]$ denote the correlation matrix of $h$ and $\boldsymbol{R}_{V}$ the correlation matrix of $h_{V}$. The two $Q P \times Q P$ correlation matrices are related by

$$
\boldsymbol{R}=\left[\tilde{A}_{T}^{*} \otimes \tilde{\boldsymbol{A}}_{R}\right] \boldsymbol{R}_{V}\left[\tilde{A}_{T}^{T} \otimes \tilde{A}_{R}^{H}\right] .
$$

Since $\left\{H_{V}(q, p)\right\}$ are approximately uncorrelated, $\boldsymbol{R}_{V}$ is always approximately diagonal. ${ }^{5}$ Furthermore, it may have some zero diagonal elements due to the sparse nature of $H_{V}$ corresponding to scattering clusters (see Fig. 8). We note that $R$ and $\boldsymbol{R}_{V}$ are also unitarily equivalent since the Kronecker product of two unitary matrices is also unitary [16].

\footnotetext{
${ }^{4}$ The approximation improves with increasing $P$ and $Q$ as $f_{Q}\left(\theta_{R}\right)$ and $f_{P}\left(\theta_{T}\right)$ in (16) become progressively peaky.

${ }^{5}$ This property of $\boldsymbol{H}_{V}$ is particularly useful in space-time code design from the viewpoint of pairwise error probability calculations [22].
} 
Using (49), we can obtain a more explicit representation for $\boldsymbol{h}$ and $\boldsymbol{R}$ in terms of the virtual representation

$$
\begin{aligned}
& \boldsymbol{h}= \sum_{q=-\tilde{Q}}^{\tilde{Q}} \sum_{p=-\tilde{P}}^{\tilde{P}} H_{V}(q, p)\left[\boldsymbol{a}_{T}^{*}\left(\tilde{\theta}_{T, p}\right) \otimes \boldsymbol{a}_{R}\left(\tilde{\theta}_{R, q}\right)\right] \\
& \boldsymbol{R} \approx \sum_{q=-\tilde{Q}}^{\tilde{Q}} \sum_{p=-\tilde{P}}^{\tilde{P}} \sigma_{q, p}^{2}\left[\boldsymbol{a}_{T}^{*}\left(\tilde{\theta}_{T, p}\right) \otimes \boldsymbol{a}_{R}\left(\tilde{\theta}_{R, q}\right)\right] \\
& \quad \times\left[\boldsymbol{a}_{T}^{T}\left(\tilde{\theta}_{T, p}\right) \otimes \boldsymbol{a}_{R}^{H}\left(\tilde{\theta}_{R, q}\right)\right]
\end{aligned}
$$

where $\sigma_{q, p}^{2}=E\left[\left|H_{V}(q, p)\right|^{2}\right]$ [see (40) and (41)]. We note that (52) is an approximate eigen-decomposition of $\boldsymbol{R}$ with the concatenated virtual basis functions $\left\{\boldsymbol{a}_{T}^{*}\left(\tilde{\theta}_{T, p}\right) \otimes \boldsymbol{a}_{R}\left(\tilde{\theta}_{R, q}\right)\right\}$ serving as the orthonormal eigenvectors and $\left\{\sigma_{q, p}^{2}\right\}$ as the corresponding eigenvalues. The corresponding Karhunen-Loeve representation relating the realizations of $\boldsymbol{H}$ and $H_{V}$ is (51).

The relation (52) between $\boldsymbol{R}$ and $\left\{\sigma_{q, p}^{2}\right\}$ provides useful insight for relating the statistics of $\boldsymbol{H}$ to the nature of physical scattering via the $k$-diagonal virtual model. Suppose that $P=Q$ for simplicity. The $\left\{\sigma_{q, p}^{2}\right\}$ in (52) represent the power of the scattering between the $p$ th virtual transmit and $q$ th virtual receive angles. The nonzero $\left\{\sigma_{q, p}^{2}\right\}$ represent the active scattering coefficients. For the $k$-diagonal model, $h_{k}$ and $\boldsymbol{R}_{k}=E\left[\boldsymbol{h}_{k} \boldsymbol{h}_{k}^{H}\right]$ take the form

$$
\begin{aligned}
& h_{k}=\sum_{p=-\tilde{P}}^{\tilde{P}} \sum_{q=\max (-\tilde{P}, p-k)}^{\min (\tilde{P}, p+k)} H_{V}(q, p) \\
& \times\left[\boldsymbol{a}_{T}^{*}\left(\tilde{\theta}_{T, p}\right) \otimes \boldsymbol{a}_{R}\left(\tilde{\theta}_{R, q}\right)\right] \\
& \boldsymbol{R}_{k}=\sum_{p=-\tilde{P}}^{\tilde{P}} \sum_{q=\max (-\tilde{P}, p-k)}^{\min (\tilde{P}, p+k)} \sigma_{q, p}^{2}\left[\boldsymbol{a}_{T}^{*}\left(\tilde{\theta}_{T, p}\right) \otimes \boldsymbol{a}_{R}\left(\tilde{\theta}_{R, q}\right)\right] \\
& \times\left[\boldsymbol{a}_{T}^{T}\left(\tilde{\theta}_{T, p}\right) \otimes \boldsymbol{a}_{R}^{H}\left(\tilde{\theta}_{R, q}\right)\right] .
\end{aligned}
$$

We note that $k=P-1$ and $\sigma_{q, p}^{2}=\sigma_{H}^{2} / P Q$ in (54) correspond to maximally rich scattering [see Figs. 6(d) and 7(b)] and yield the extreme case of iid $H\left(\boldsymbol{R}_{P-1}=\boldsymbol{I}\right)$. For diagonal virtual scattering [see Figs. 6(c) and 7(a))], $k=0$ in (54), $\sigma_{q, p}^{2}$ is nonzero only for $p=q$, and there is significant correlation between elements of $\boldsymbol{H}$. As demonstrated in Section V, this approach for modeling progressively rich scattering (progressively higher diversity) via a $k$-diagonal virtual model provides a direct link between the nature of scattering in each cluster and the statistics induced by it, which is very insightful in capacity calculations. In particular, $\boldsymbol{H}_{0}$ (diagonal) and $\boldsymbol{H}_{P-1}$ (iid) have nearly identical ergodic capacities under appropriate power normalization but significantly different outage capacities due to higher diversity in $\boldsymbol{H}_{P-1}$.

\section{EfFect of Antenna Spacing: Spatial ZOOMING AND ALIASING}

For any given scattering environment, antenna spacing can have a marked effect on capacity and diversity. It is well known that increasing antenna spacing can decorrelate the elements of
$H$. In this section, we use the virtual channel representation to provide a simple and intuitive explanation for the effect of antenna spacing on channel statistics, diversity, and capacity. In particular, we show that increasing antenna spacing not only decorrelates the channel (and thus partially increases capacity as well) but also directly contributes to increased capacity by effectively increasing the number of parallel channels. Capacity of each cluster depends on both the size of the corresponding sub-matrix of $\boldsymbol{H}_{V}$ as well as the number of nonvanishing entries within it (captured by $k$-diagonal modeling, for example). Antenna spacing contributes to both factors. The key to this understanding is (4) relating the spatial variables $\theta$ and $\phi$ and the notion of spatial aliasing for $\alpha>0.5$, as illustrated in Fig. 1.

For simplicity of exposition, assume that $\alpha_{T}=\alpha_{R}=\alpha$. Consider a square scattering cluster with angular spreads in the $\phi$ domain given by $S_{R}=\left[-\Delta \phi_{R}, \Delta \phi_{R}\right]$ and $S_{T}=\left[-\Delta \phi_{T}, \Delta \phi_{T}\right]$. That is, the spreading function in the $\phi$ domain $G_{o}\left(\phi_{R}, \phi_{T}\right)=G\left(\alpha \sin \left(\phi_{R}\right), \alpha \sin \left(\phi_{T}\right)\right)$ is nonzero only for $\left(\phi_{T}, \phi_{R}\right) \in S_{T} \times S_{R}$. Fig. 9 shows one such cluster with $\Delta \phi_{R}=\Delta \phi_{T}=\pi / 8$. The essential effect of increasing antenna spacing $\alpha$ is spatial zooming, as illustrated in Fig. 9; the antenna array is able to zoom into the scattering cluster in that $\left\{\left(\phi_{T}, \phi_{R}\right) \in\left[-\Delta \phi_{T}, \Delta \phi_{T}\right] \times\left[-\Delta \phi_{R}, \Delta \phi_{R}\right]\right\}$ maps to $\left\{\left(\theta_{T}, \theta_{R}\right) \in\left[-\alpha \sin \left(\Delta \phi_{T}\right), \alpha \sin \left(\Delta \phi_{T}\right)\right] \times\left[-\alpha \sin \left(\Delta \phi_{R}\right)\right.\right.$, $\left.\left.\alpha \sin \left(\Delta \phi_{R}\right)\right]\right\}$ in the $\theta$ domain. ${ }^{6}$ As illustrated in Fig. 9, the small cluster in the $\phi$ domain occupies an increasingly larger portion of the $\theta$ range as $\alpha$ increases. In particular, the cluster occupies the entire region for $\alpha=1.31$.

Another effect intimately related to spatial zooming is spatial aliasing. When the edges of the cluster (edges of $G\left(\theta_{R}, \theta_{T}\right)$ ) exceed the principal $\theta$ range as $\alpha$ increases, they alias back into the principal range as captured by $\hat{G}\left(\theta_{R}, \theta_{T}\right)$ in (8) and (11). This effect is illustrated in Fig. 10 for four clusters with identical widths in the $\theta$ domain. For $\alpha=0.5$, all clusters have $\theta$ widths of $1 / 8 \times 1 / 8$, as in Fig. 10(a). Doubling the spacing to $\alpha=1$ doubles the effective size of the clusters in the $\theta$ domain and scales their centers as well, as shown in Fig. 10(b). Finally, another doubling of spacing to $\alpha=2$ results in clusters occupying the entire $\theta$ range, as in Fig. 10(c). Spatial aliasing of clusters 1,3 , and 4 is crucial to their covering the entire $\theta$ region at $\alpha=2$. Considering the size of any one cluster, we would expect an eight-fold increase in spacing for the clusters to occupy the entire $\theta$ region. However, due to spatial aliasing, only a four-fold increase is needed.

The effect of antenna spacing on capacity is revealed by $\boldsymbol{H}_{V}$ via (3), which states that $\left\{H_{V}(q, p)\right\}$ are uniformly spaced samples of $\hat{G}\left(\theta_{R}, \theta_{T}\right)$ corresponding to the virtual angles. Thus, as $\alpha$ increases, increasingly more virtual angles couple with the scatterers, resulting in increasingly more nonvanishing (and approximately uncorrelated) $\left\{H_{V}(q, p)\right\}$, thereby increasing the rank of $\boldsymbol{H}_{V}$ and, hence, capacity. Note that this increase in capacity happens without an increase in channel power or received power in the case of a discrete channel [see (35)]. Similarly,

${ }^{6}$ In effect, the scattering cluster acts as a magnifying glass, providing a magnified image of the transmitter array at the receiver and vice versa [1]. The magnification increases with $\alpha$ and makes it possible for the receiver to resolve the signals from different transmitter elements, which would not be possible without scattering. 


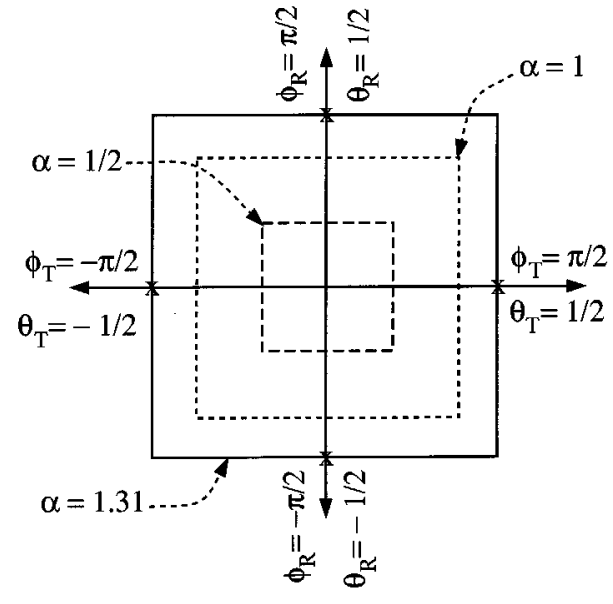

Fig. 9. Schematic illustrating the effect of antenna spacing in a single-cluster environment. The cluster has support $[-\pi / 8, \pi / 8] \times[-\pi / 8, \pi / 8]$ in the $\phi$ domain, which corresponds to $[-0.19,0.19] \times[-0.19,0.19]$ in the $\theta$ domain for $\alpha=0.5$. Doubling the spacing to $\alpha=1$ zooms into the cluster and doubles its $\theta$ support. Finally, the cluster covers the entire $\theta$ region for $\alpha=1.31$.

from (42) and (48), we conclude that $\{H(m, n)\}$ decorrelate with increased antenna spacing since the supports of $\hat{M}\left(\theta_{R}, \theta_{T}\right)$ and $\boldsymbol{H}_{V}$ increase. This effect also contributes to higher diversity and capacity. Interestingly, we find that spatial aliasing, which is considered an undesirable effect in traditionally array processing applications, plays a positive role in increasing capacity!? In Section VI, we discuss capacity issues in more detail and present numerical results to quantify the effects of antenna spacing on capacity associated with the environments in Figs. 9 and 10 .

\section{CAPacity CALCUlations}

In this section, we illustrate the ease of computation and simple interpretation afforded by virtual channel representation for capacity calculations. Shannon capacity of a fading channel observed over a finite duration is strictly zero. Thus, our focus here is on outage capacity [18], which is a metric that is more appropriate to fading channels. Outage capacity reflects the maximum rate that can be guaranteed with a certain probability. We first discuss two key parameters that control capacity and relate them to scattering characteristics.

\section{A. Parallel Channels and Diversity}

The "image" of the scattering environment provided by $\boldsymbol{H}_{V}$ is intimately related to two key channel parameters from a communication theoretic viewpoint: the number of parallel chan$n e l s, P C$, that primarily controls capacity, and the level of $d i$ versity per parallel channel, $D$, that primarily controls the slope of the error probability curves as well as the slope of outage capacity curves. These two factors are evident in the lower bound for capacity, conditioned on a channel realization, obtained by Foschini et al. [1] in their derivation of the BLAST architecture for a system with $P=Q$ antennas under rich scattering (iid $\boldsymbol{H}$ )

$$
C(H)>\sum_{k=1}^{P} \log _{2}\left(1+\left(\frac{\rho}{P}\right) \chi_{2 k}^{2}\right) \text { bits } / \mathrm{s} / \mathrm{Hz}
$$

${ }^{7}$ We thank one of the reviewers for this observation.

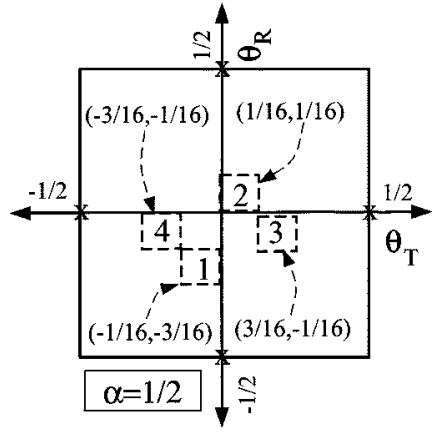

(a)

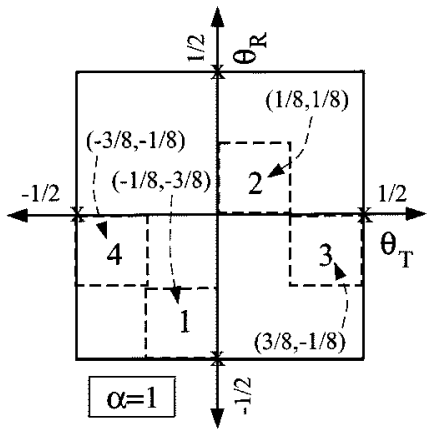

(b)

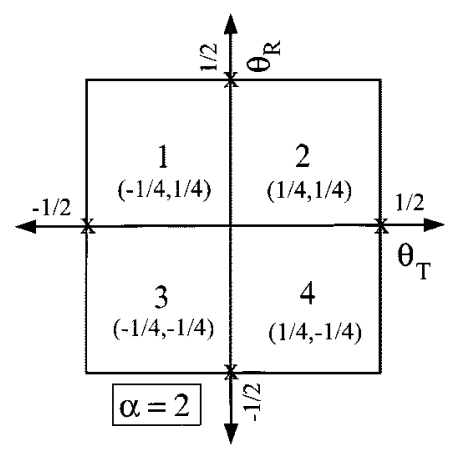

(c)

Fig. 10. Schematic illustrating the effect of antenna spacing on a four-cluster environment. The clusters have identical $(1 / 8 \times 1 / 8$-wide) supports in the $\theta$ domain for $\alpha=0.5$, as depicted in (a). For $\alpha=1$, the support of the clusters doubles and their centers get scaled relative to $\alpha=0.5$, as in (b). Doubling the antenna spacing again to $\alpha=2$ makes the clusters occupy the entire $\theta$ range, as in (c). Clusters 1,3 , and 4 undergo spatial aliasing at $\alpha=2$. For example, the center of cluster 3 scales to $(3 / 4,-1 / 4)$, which gets aliased into the principle range to $(-1 / 4,-1 / 4)$.

where $\chi_{2 k}^{2}$ denotes a chi-squared random variable with $2 k$ degrees of freedom. The above relation states that the multiantenna channel can be decomposed into $P C=P$ parallel channels with varying levels of diversity $(D=k)$ captured by $\chi_{2 k}^{2}$. $P C$ quantifies the linear multiplier in capacity afforded by multiple antennas, and $D$ quantifies the stabilization of the random SNR associated with each parallel channel. $P C \leq P C_{\max }=$ $\min (P, Q)$, and the virtual path partitioning tells us that there have to be at least $L=\min (P, Q)$ strictly distinct physical paths corresponding to distinct virtual spatial bins (27) to achieve $P C_{\max }$. However, this does not guarantee maximum diversity for each parallel channel. An example of this situation is "diagonal" scattering $\left[\boldsymbol{H}_{0}\right.$ in (28)] corresponding to Fig. 7(a) that achieves $P C_{\max }$ but $D=1 . D \leq D_{\max }=\max (P, Q)$ and if $P \leq Q$, each virtual transmit angle must couple with $D_{\max }=$ $Q$ distinct virtual receive angles via distinct paths in order to achieve $D_{\max }$. This corresponds to receive diversity. On the other hand, if $P \geq Q, D_{\max }=P$ distinct virtual transmit angles must couple to each virtual receive angle via distinct paths in order to achieve $D_{\max }$. This corresponds to transmit diversity. Thus, we need at least $\min (P, Q) \times \max (P, Q)=P Q$ strictly distinct paths, corresponding to distinct virtual spatial bins (27) to excite all the degrees of freedom in the channel and achieve both $P C_{\max }$ and $D_{\max }$. This corresponds to maximally rich scattering [ $\boldsymbol{H}_{P-1}$ in (28)], corresponding to Fig. 7(b).

We note that the requirement for the paths to be distinct (for diversity) and strictly distinct (for parallel channels) is impor- 
tant. Consider a $5 \times 5$ array and five propagation paths for illustration. If the paths are distinct only in the transmit direction, they can only be exploited for five-level transmit diversity. If they are distinct only in the receive direction, they can only be exploited for full receive diversity. However, in both above cases, the paths only contribute to a single parallel channel $\left(P C=1\right.$ and $\left.D=D_{\max }=5\right)$. If the paths are strictly distinct, they would contribute to five parallel channels that could be exploited for maximum capacity $\left(P C_{\max }=5\right.$, $D=1)$ or maximum transmit/receive diversity $(P C=1$, $\left.D_{\max }=5\right)$ or a combination thereof $\left(P C<P C_{\max }, D<\right.$ $\left.D_{\max }\right)$. As an extreme example, if all paths are confined to a single virtual spatial bin (27), $P C$ and $D$ will be close to 1 no matter how many paths there are! Achieving $P C_{\max }$ and $D_{\max }$ requires maximum scattering spreads and maximally rich scattering for $\alpha=0.5$. Smaller spreads and less rich scattering result in lower values of $P C$ and $D$ for $\alpha=0.5$ but can yield maximum values by sufficiently increasing $\alpha$. This is due to spatial zooming and aliasing, which results in more virtual angles coupling to the scatterers.

\section{B. Capacity Expressions}

For simplicity, let $P=Q$. Consider the noisy channel $\boldsymbol{x}=$ $\sqrt{\rho} \boldsymbol{H} \boldsymbol{s}+\boldsymbol{w}$, where $\rho$ is the transmitted power $\left.\left(E\|\boldsymbol{s}\|^{2}\right]=1\right)$, and $\boldsymbol{w}$ is zero-mean complex Gaussian noise vector with $E\left[\boldsymbol{w} \boldsymbol{w}^{H}\right]=$ $\boldsymbol{I}$. Conditioned on the knowledge of $\boldsymbol{H}$ (or $\boldsymbol{H}_{V}$ ) at the receiver, channel capacity is approximately ${ }^{8}$ given by [1], [2]

$$
C(\boldsymbol{H}) \approx \log _{2}\left[\operatorname{det}\left(\boldsymbol{I}+\frac{\rho \boldsymbol{H}_{V} \boldsymbol{H}_{V}^{H}}{P}\right)\right] \text { bits/s/Hz }
$$

where we have used unitary equivalence of $\boldsymbol{H}$ and $\boldsymbol{H}_{V}$. The ergodic capacity is given by $C_{E}=E\left[C\left(\boldsymbol{H}_{V}\right)\right]$, where the expectation is over the statistics of $\boldsymbol{H}_{V}$. For a clustered scattering en-

\footnotetext{
${ }^{8}$ The expression in (56) is an approximation since it strictly applies to full iid $\boldsymbol{H}$ or $\boldsymbol{H}_{V}$. A closed-form expression for the exact capacity of a sparse $\boldsymbol{H}_{V}$ is an open problem. Nevertheless, (56) serves as a useful approximation for $H_{V}$ consisting of smaller nonvanishing sub-matrices with uncorrelated (and in some cases iid) elements. A detailed discussion of this technical issue is beyond the scope of this paper and will be reported elsewhere.
}

vironment, the corresponding decomposition of $\boldsymbol{H}_{V}$ (see Fig. 8) can be used to induce an approximate decomposition of (56). Fig. 8 strictly applies to an environment in which the clusters (and the corresponding sub-matrices) are strictly distinct, that is, they have disjoint supports in both transmit and receive angles. In general, some clusters may overlap on either the receive or transmit side, in which case, the overlapping clusters will contribute to additional diversity as well as interference. For example, consider $\boldsymbol{H}_{V}$ of the form

$$
\boldsymbol{H}_{V}=\left[\begin{array}{ccc}
\boldsymbol{H}_{V}(1) & \boldsymbol{H}_{V}(2) & \mathbf{0} \\
\mathbf{0} & \boldsymbol{H}_{V}(3) & \mathbf{0} \\
\boldsymbol{H}_{V}(4) & \mathbf{0} & \boldsymbol{H}_{V}(5)
\end{array}\right]
$$

corresponding to five clusters. Strictly distinct clusters correspond to $\boldsymbol{H}_{V}(1), \boldsymbol{H}_{V}(3)$, and $\boldsymbol{H}_{V}(5)$. The sets of clusters $\left\{\boldsymbol{H}_{V}(1), \boldsymbol{H}_{V}(2)\right\},\left\{\boldsymbol{H}_{V}(3)\right\}$, and $\left\{\boldsymbol{H}_{V}(4), \boldsymbol{H}_{V}(5)\right\}$ are distinct in receive angles, whereas the sets of clusters $\left\{\boldsymbol{H}_{V}(1), \boldsymbol{H}_{V}(4)\right\},\left\{\boldsymbol{H}_{V}(2), \boldsymbol{H}_{V}(3)\right\}$, and $\left\{\boldsymbol{H}_{V}(5)\right\}$ are distinct in transmit angles. The capacity is governed by $\boldsymbol{H}_{V} \boldsymbol{H}_{V}^{H}$ or $\boldsymbol{H}_{V}^{H} \boldsymbol{H}_{V}$, where the first form is useful for distinguishing clusters in receive angles, and the second form for distinguishing them in transmit angles. In this case, we have (58) and (59), shown at the bottom of the page. The diagonal blocks correspond to sets of distinct clusters ${ }^{9}$ that mainly contribute to parallel channels and diversity, whereas the off-diagonal blocks represent the interference between them. However, since the different cluster sub-matrices are uncorrelated, the diagonal blocks dominate, especially as the size of the sub-matrices increases. Thus, when the number of antennas is large, we have the approximate decompositions for (56), shown in (60) and (61) at the bottom of the page, where $N_{c}$ is the number of strictly distinct clusters, (60) distinguishes clusters in receive angle, and (61) distinguishes them in transmit angles. In (60), $N_{R, i}$ is the number of clusters that overlap in receive angles with the $i$ th strictly distinct cluster, and $\left\{\boldsymbol{H}_{V, R}(i, j)\right\}$ are the corresponding sub-matrices. For example, in (57), $N_{R, 1}=2$, $\boldsymbol{H}_{V, R}(1,1)=\boldsymbol{H}_{V}(1)$, and $\boldsymbol{H}_{V, R}(1,2)=\boldsymbol{H}_{V}(2)$. Similarly, in (61), $N_{T, i}$ is the number of clusters that overlap in transmit

${ }^{9}$ Distinct in receive angles in (58) and in transmit angles in (59).

$$
\begin{aligned}
\boldsymbol{H}_{V} \boldsymbol{H}_{V}^{H} & =\left[\begin{array}{ccc}
\boldsymbol{H}_{V}(1) \boldsymbol{H}_{V}^{H}(1)+\boldsymbol{H}_{V}(2) \boldsymbol{H}_{V}^{H}(2) & \boldsymbol{H}_{V}(2) \boldsymbol{H}_{V}^{H}(3) & \boldsymbol{H}_{V}(1) \boldsymbol{H}_{V}^{H}(4) \\
\boldsymbol{H}_{V}(3) \boldsymbol{H}_{V}^{H}(2) & \boldsymbol{H}_{V}(3) \boldsymbol{H}_{V}^{H}(3) & 0 \\
\boldsymbol{H}_{V}(4) \boldsymbol{H}_{V}^{H}(1) & \mathbf{0} & \boldsymbol{H}_{V}(4) \boldsymbol{H}_{V}^{H}(4)+\boldsymbol{H}_{V}(5) \boldsymbol{H}_{V}^{H}(5)
\end{array}\right] \\
\boldsymbol{H}_{V}^{H} \boldsymbol{H}_{V}= & {\left[\begin{array}{ccc}
\boldsymbol{H}_{V}^{H}(1) \boldsymbol{H}_{V}(1)+\boldsymbol{H}_{V}^{H}(4) \boldsymbol{H}_{V}(4) & \boldsymbol{H}_{V}^{H}(1) \boldsymbol{H}_{V}(2) & \boldsymbol{H}_{V}^{H}(4) \boldsymbol{H}_{V}(5) \\
\boldsymbol{H}_{V}^{H}(2) \boldsymbol{H}_{V}(1) & \boldsymbol{H}_{V}^{H}(2) \boldsymbol{H}_{V}(2)+\boldsymbol{H}_{V}^{H}(3) \boldsymbol{H}_{V}(3) & \mathbf{0} \\
\boldsymbol{H}_{V}^{H}(5) \boldsymbol{H}_{V}(4) & \mathbf{0} & \boldsymbol{H}_{V}^{H}(5) \boldsymbol{H}_{V}(5)
\end{array}\right] . }
\end{aligned}
$$

$$
\begin{aligned}
& C(\boldsymbol{H}) \approx \sum_{i=1}^{N_{c}} \log _{2}\left[\operatorname{det}\left(\boldsymbol{I}+\frac{\rho}{P} \sum_{j=1}^{N_{R, i}} \boldsymbol{H}_{V, R}(i, j) \boldsymbol{H}_{V, R}^{H}(i, j)\right)\right] \mathrm{bits} / \mathrm{s} / \mathrm{Hz} \\
& C(\boldsymbol{H}) \approx \sum_{i=1}^{N_{c}} \log _{2}\left[\operatorname{det}\left(\boldsymbol{I}+\frac{\rho}{P} \sum_{j=1}^{N_{T, i}} \boldsymbol{H}_{V, T}^{H}(i, j) \boldsymbol{H}_{V, T}(i, j)\right)\right] \mathrm{bits} / \mathrm{s} / \mathrm{Hz}
\end{aligned}
$$


angles with the $i$ th strictly distinct cluster, and $\left\{\boldsymbol{H}_{V, T}(i, j)\right\}$ are the corresponding sub-matrices. In (57), $N_{T, 1}=2$, $\boldsymbol{H}_{V, T}(1,1)=\boldsymbol{H}_{V}(1)$, and $\boldsymbol{H}_{V, T}(1,2)=\boldsymbol{H}_{V}(4)$. The above interpretation of the capacity of an arbitrary spatial channel in terms of $\boldsymbol{H}_{V}$ can be summarized as follows.

A spatial channel corresponding to an arbitrary clustered scattering environment represented by $\mathrm{H}_{V}$ can be decomposed into $N_{c}$ independent virtual spatial sub-channels corresponding to strictly distinct clusters. The number of parallel channels $(P C)$ and diversity per parallel channel $(D)$ in each sub-channel are contributed by both the strictly distinct clusters as well as clusters that overlap with them in receive or transmit virtual angles as exemplified by (60) and (61), respectively. Within each sub-channel, PC is determined by the sizes of the constituent cluster sub-matrices, and $D$ is determined by the nature of scattering in the clusters that can be captured by a $k$-diagonal model (with uncorrelated entries) for the sub-matrices.

\section{Numerical Examples}

We now present some numerical results to illustrate various aspects of the virtual representation framework. Our illustrations are in the context of both an idealized rich scattering environment and more realistic environments consisting of scattering clusters with smaller angular spreads. In all cases, $P=$ $Q=11$ and SNR $=10 \log _{10}(\rho)=20 \mathrm{~dB}(\rho=100)$. The iid channel matrices have unit variance complex Gaussian entries, resulting in $\sigma_{H}^{2}=P^{2}=121$. In clustered scattering environments, each cluster is simulated via the physical model (9) using $L=200$ paths. The channel power is equally distributed between the clusters. Within each cluster, each path is associated with random $\left(\phi_{T}, \phi_{R}\right)$ that are uniformly distributed within the angular spreads of the cluster. The path gains are simulated as iid zero-mean complex Gaussian random variables with power $\sigma^{2}$ adjusted so that $L \sigma^{2}$ equals the total power associated with that cluster $\left(\sigma_{H}^{2} / 4\right.$ for the environment in Fig. 10). The outage capacity plots are computed from 1000 independent channel realizations.

Fig. 11 compares the capacity of $k$-diagonal approximations to the true channel matrix in both an idealized rich scattering environment and a more realistic environment consisting of a limited spread cluster. In the iid case, which is depicted in Fig. 11(a), $\boldsymbol{H}_{V}$ for computing $\boldsymbol{H}_{k}$ in (28) is computed from $\boldsymbol{H}$ via (15) with $\alpha_{T}=\alpha_{R}=0.5$. The matrix $\boldsymbol{H}_{k}$ is scaled so that the received SNR is the same in all cases. The scaling factor is given by $\sqrt{P^{2} /(P+k(2 P-k-1))}, k=0, \ldots, P-1$, which equals $\sqrt{P}$ for $k=0$ (diagonal approximation) and 1 for $k=P-1$ (full matrix). As evident from Fig. 11(a), the outage capacity curve is much steeper for the full iid matrix compared with the diagonal approximation due to higher diversity in the former case. Furthermore, the performance of a three-diagonal approximation is fairly close to the iid channel (ten-diagonal), demonstrating that for the same received SNR, the three-diagonal system captures most of the diversity advantage. The ergodic capacities are 60.4, 65, and $60 \mathrm{bits} / \mathrm{s} / \mathrm{Hz}$ for the iid, diagonal, and three-diagonal channels, respectively. Note that the diagonal channel yields a slightly higher ergodic capacity, even though its outage capacity performance is worse.

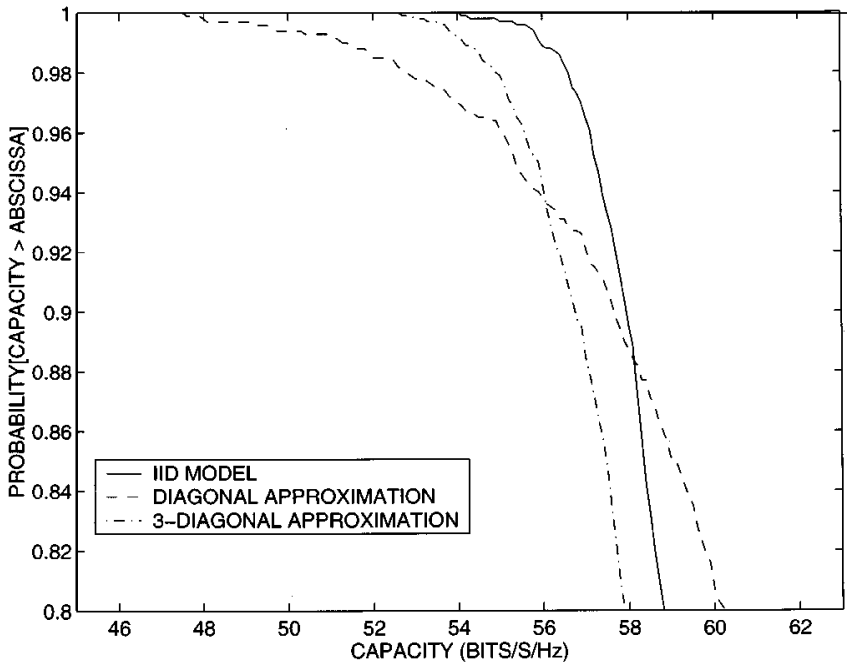

(a)

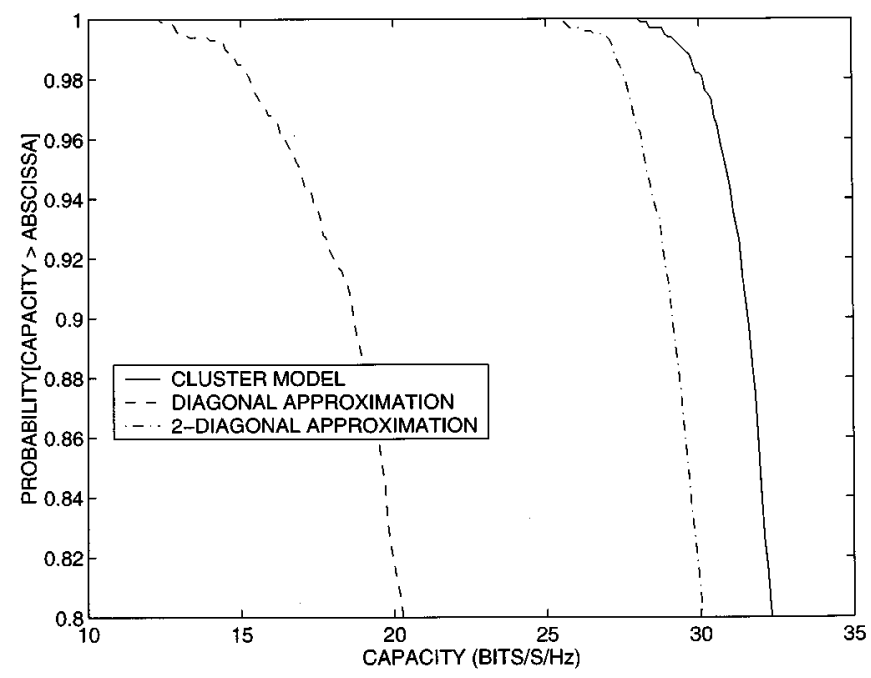

(b)

Fig. 11. Capacity comparison of $k$-diagonal approximations. (a) Maximally rich (iid) scattering environment. The $k$-diagonal approximations are scaled so that all matrices have same average power-the level of diversity is the main difference between the approximations. (b) Realistic environment consisting of a $\pi / 4 \times \pi / 4$-wide cluster centered at the origin. The channel power is not normalized in this case. Both the level of diversity and received SNR contribute to differences in performance.

This may be attributed to the lack of interference between the parallel channels in the diagonal case.

Fig. 11(b) compares the capacity of $k$-diagonal approximations for a $\pi / 4 \times \pi / 4$-wide cluster centered at $\left(\phi_{T}, \phi_{R}\right)=$ $(0,0)$. The channel power is not normalized in this case; the powers are 121.6, 23.3, and 93.3 for the full, diagonal, and two-diagonal approximations. As evident, the capacity of the two-diagonal approximation is fairly close to that of the full iid channel. This is because $P C$ and $D$ are relatively small in this case due to limited scattering spread. The ergodic capacities are $33.8,23.2$, and $31.8 \mathrm{bits} / \mathrm{s} / \mathrm{Hz}$ for the full, diagonal, and two-diagonal approximations, respectively, and the differences in capacities also reflect differences in received SNR in addition to diversity.

We now illustrate the effect of antenna spacing on capacity by simulating channels corresponding to the scattering environ- 


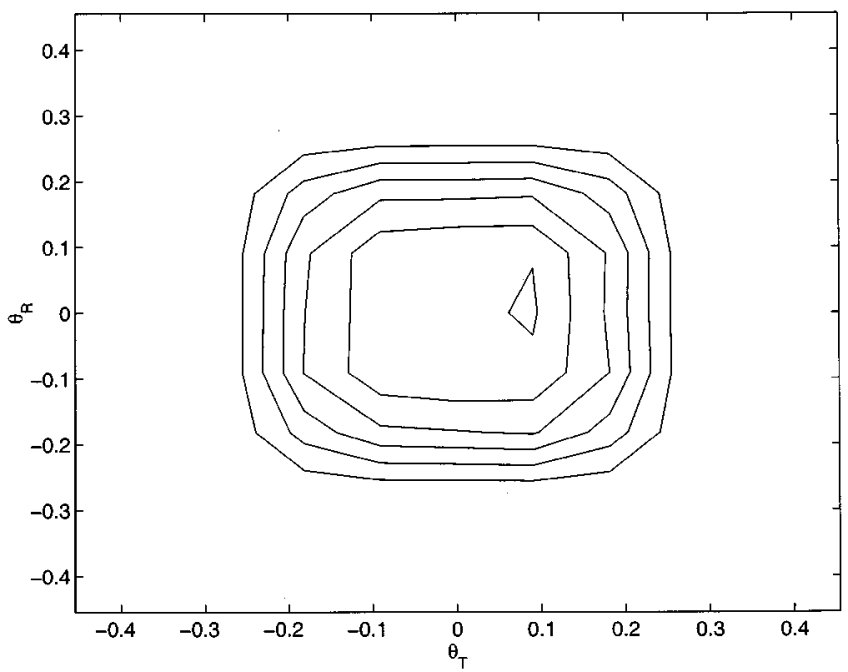

(a)

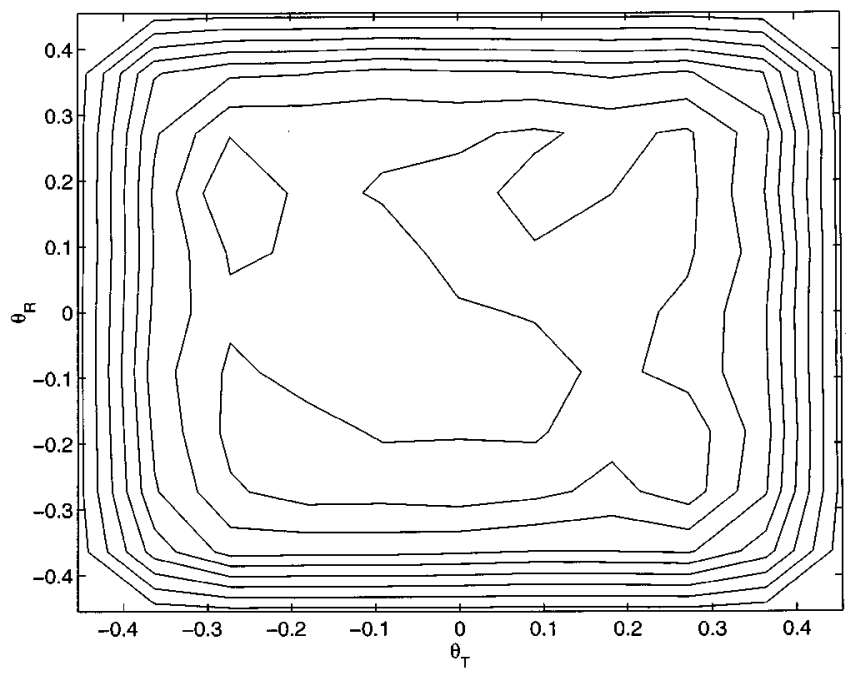

(c)

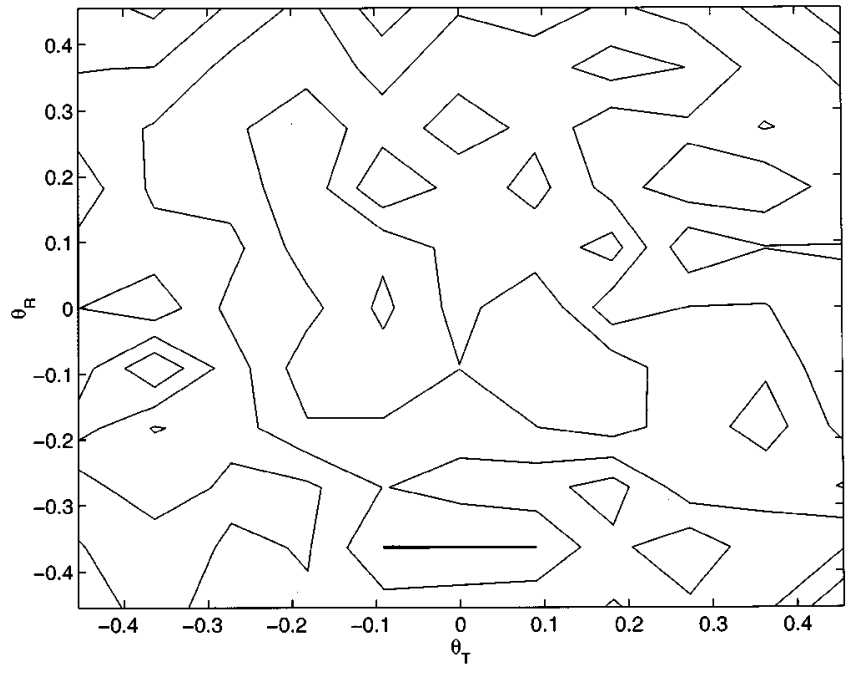

(e)

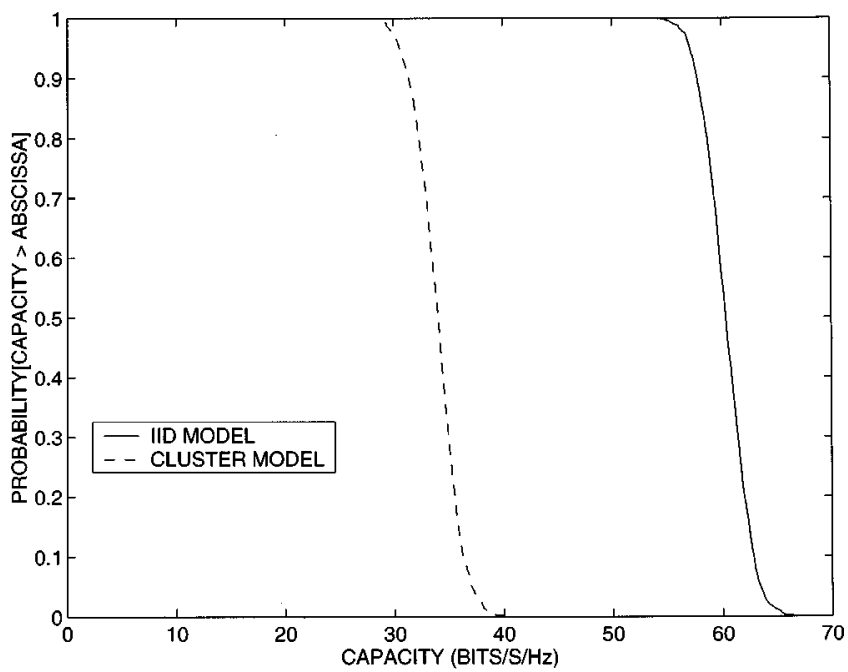

(b)

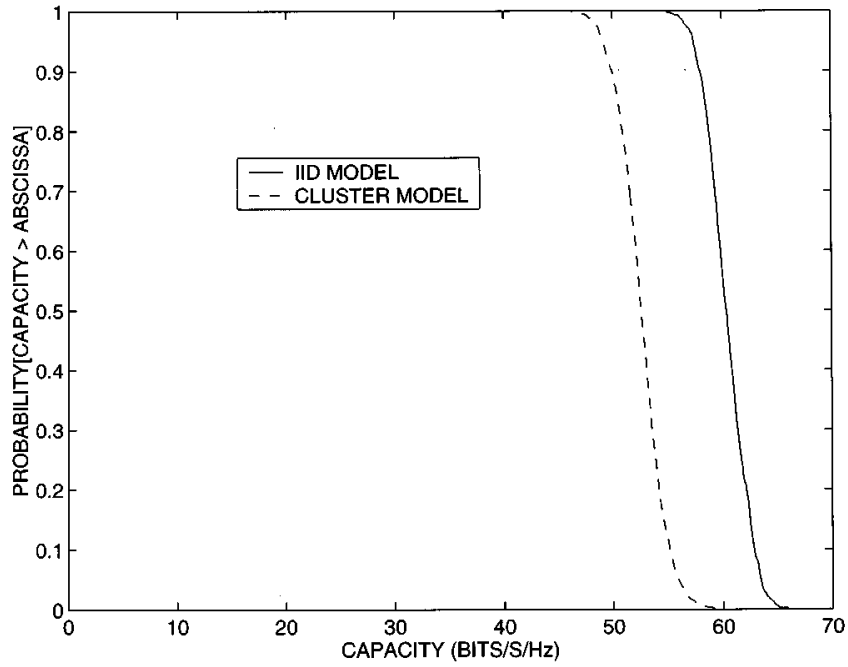

(d)

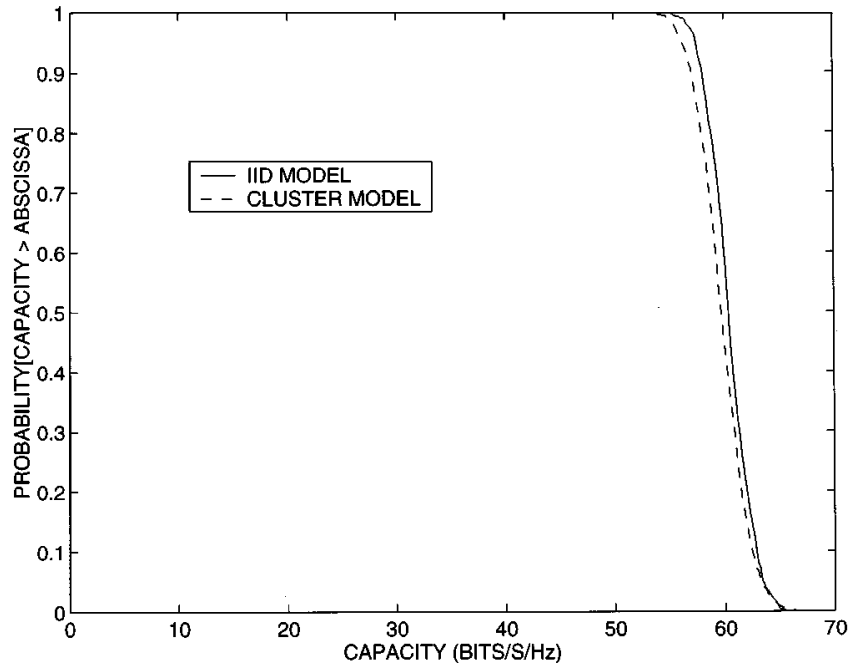

(f)

Fig. 12. Effect of antenna spacing on capacity for the single-cluster environment depicted in Fig. 9. Contour plots of $E\left[\left|H_{V}(q, p)\right|^{2}\right]$ and outage capacity curves for both the clustered channel and an iid channel are shown. (a) Contour plot of $E\left[\left|H_{V}(p, q)\right|^{2}\right]$ for $\alpha=0.5$. (b) Outage capacity plots for $\alpha=0.5$. (c) and (d) correspond to (a) and (b) for $\alpha=1.0$ and (e) and (f) correspond to $\alpha=1.31$. Note that the support of $E\left[\left|H_{V}(p, q)\right|^{2}\right]$ closely matches the supports in Fig. 9. For $\alpha=1.31$ the clustered channel has the same capacity as the iid channel due to maximum zooming. 


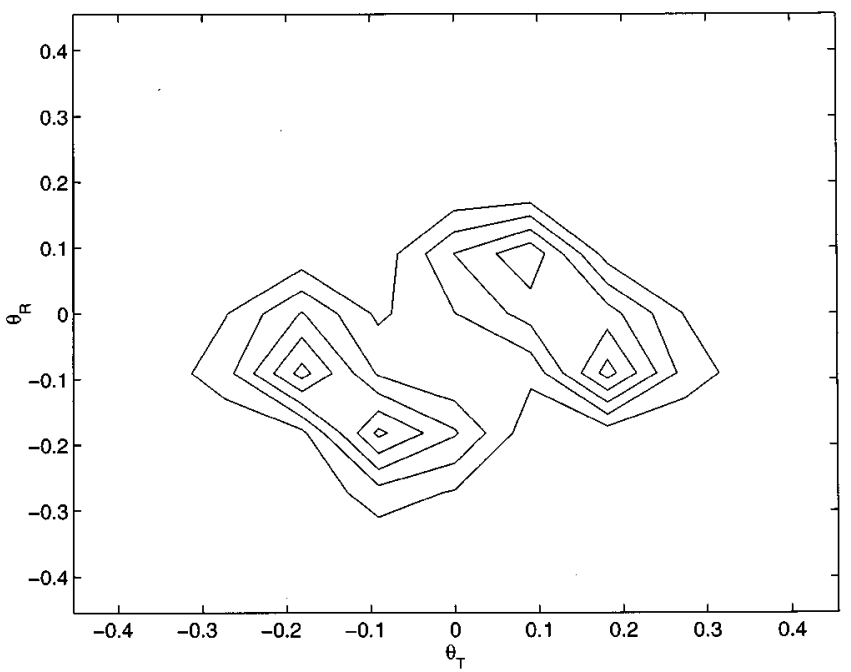

(a)

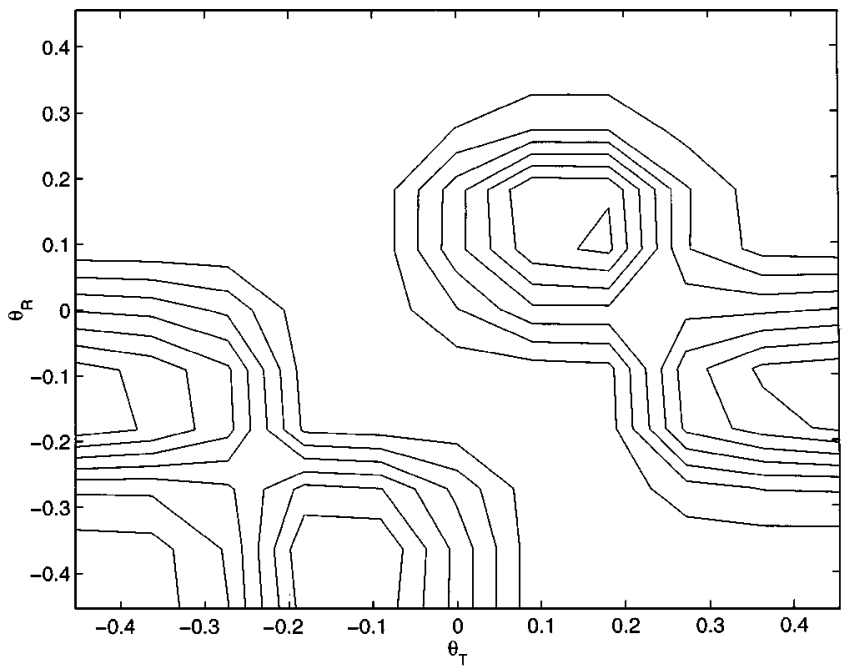

(c)

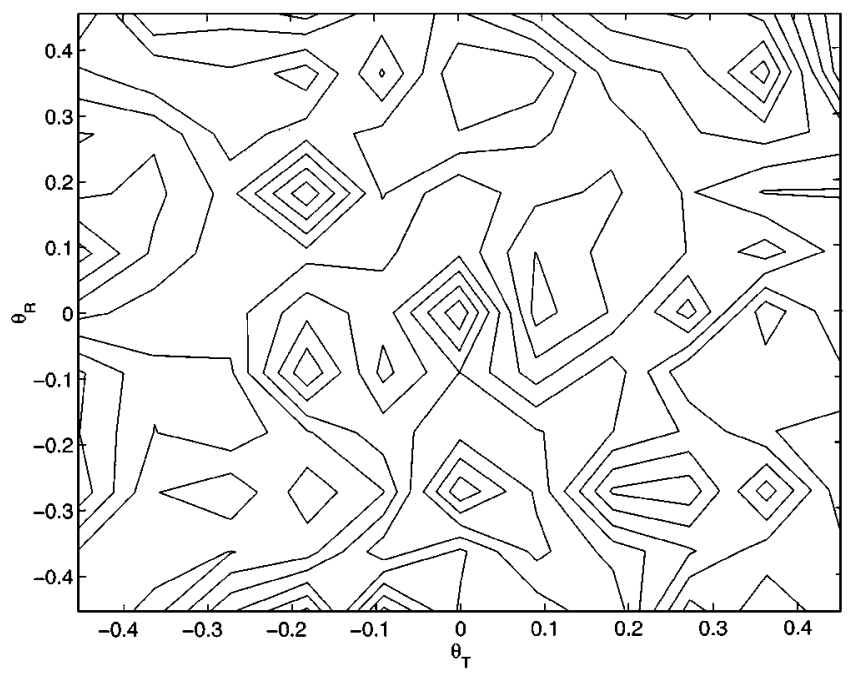

(e)

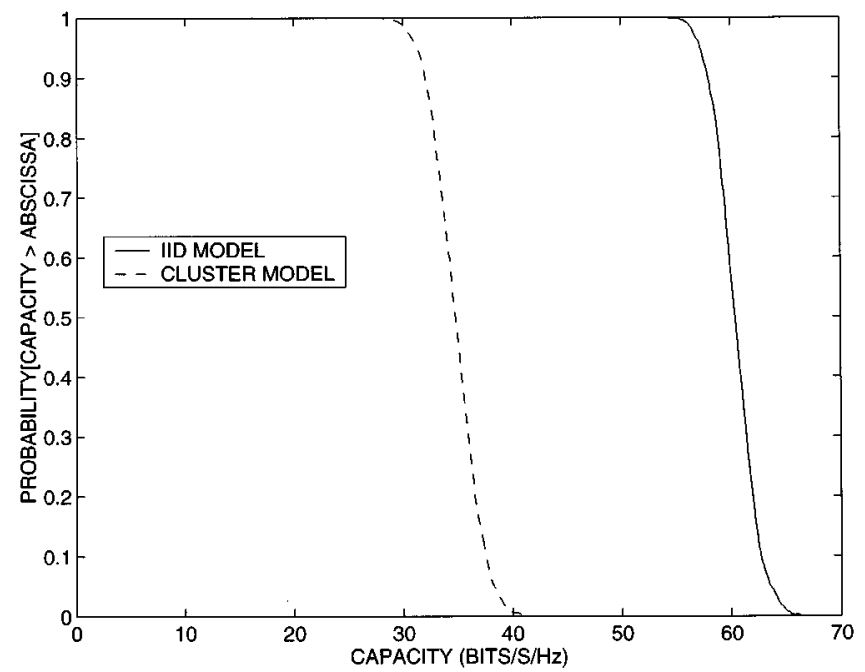

(b)

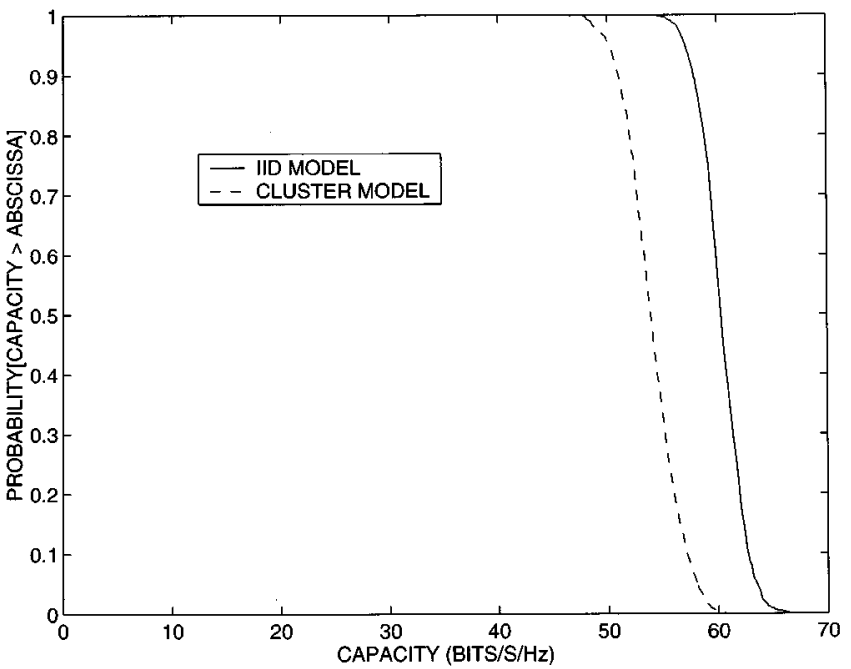

(d)

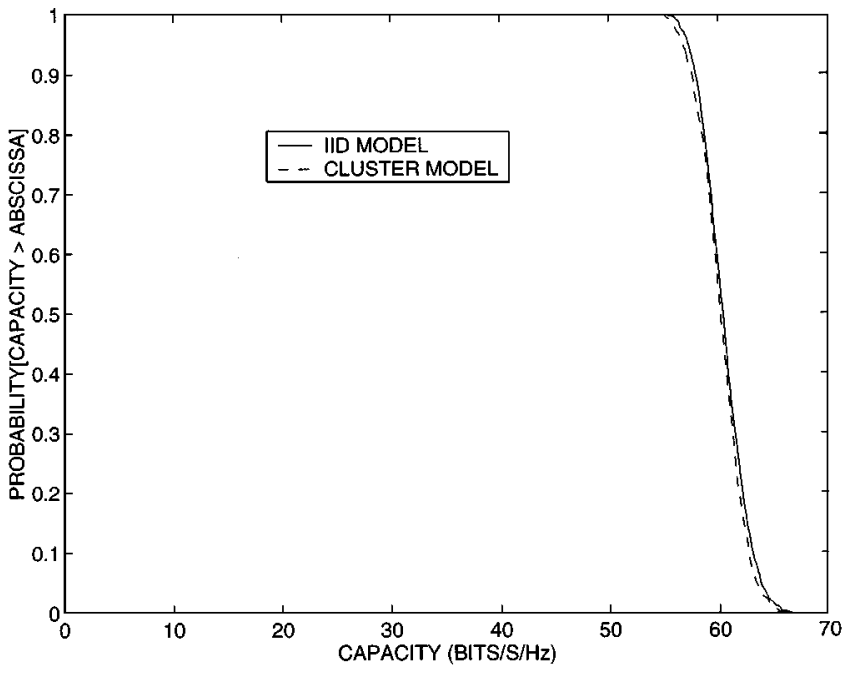

(f)

Fig. 13. Effect of antenna spacing on capacity for the four-cluster environment in Fig. 10. Contour plots of $E\left[\left|H_{V}(q, p)\right|^{2}\right]$ and outage capacity curves for both the clustered channel and an iid channel are shown. (a) Contour plot of $E\left[\left|H_{V}(p, q)\right|^{2}\right]$ for $\alpha=0.5$. (b) Outage capacity plots for $\alpha=0.5$. (c) and (d) correspond to (a) and (b) for $\alpha=1.0$ and (e) and (f) correspond to $\alpha=2.0$. Note that the support of $E\left[\left|H_{V}(p, q)\right|^{2}\right]$ closely match the supports in Fig. 10 . For $\alpha=2.0$ the clustered channel has the same capacity as the iid channel due to spatial zooming and aliasing. 
ments depicted in Figs. 9 and 10. We compare the outage capacity of the clustered channel to that of an iid channel with the same $\rho$. As the examples in Figs. 12 and 13 demonstrate, any scattering environment with limited angular spreads can yield an iid channel matrix via the zooming and aliasing effects by increasing antenna spacing.

Fig. 12 corresponds to the single-cluster environment depicted in Fig. 9. Two quantities are plotted for three antenna spacings $\alpha=0.5,1.0$, and 1.31. First, a contour plot of $\left.E\left[\left|H_{V}(p, q)\right|\right]^{2}\right]$ is provided to show the support of $\hat{M}\left(\theta_{R}, \theta_{T}\right)$ and the effect of spatial zooming on it. Second, an outage capacity plot is provided along with the outage capacity of an iid channel (with the same $\rho$ ) for comparison. As evident from Figs. 12(a), (c) and (e), the effective size of the cluster in the $\theta$ domain increases due to spatial zooming as $\alpha$ increases. This is accompanied by a corresponding increase in capacity due to increase in the number of parallel channels and decorrelation of channel coefficients as increasingly more virtual angles couple with the scatterers. We note that the ergodic capacity of the iid channel is $60.4 \mathrm{bits} / \mathrm{s} / \mathrm{Hz}$, and that of the clustered channel is 33.9, 52.6, and $59.8 \mathrm{bits} / \mathrm{s} / \mathrm{Hz}$ for $\alpha=0.5,1.0$ and 1.31. Recall from Fig. 9 that for $\alpha=1.31$, the cluster covers the entire $\theta$ region, thereby effectively yielding an iid channel. This is confirmed by Fig. 12(e) and (f).

Fig. 13 illustrates the effect of antenna spacing on capacity for the four-cluster environment depicted in Fig. 10. Again, contour plots of $\left.E\left[\left|H_{V}(p, q)\right|\right]^{2}\right]$ and outage capacity plots are shown for $\alpha=0.5,1.0$, and 2.0. Fig. 13(a), (c), and (e) corresponds to Fig. 10(a)-(c), respectively. The ergodic capacity of the iid channel is $60.4 \mathrm{bits} / \mathrm{s} / \mathrm{Hz}$, and that of the clustered channel is 34.8, 53.9, and $60.2 \mathrm{bits} / \mathrm{s} / \mathrm{Hz}$ for $\alpha=0.5,1.0$ and 2.0. Recall from Fig. 10(c) that for $\alpha=2.0$, the clusters cover the entire $\theta$ region due to spatial zooming and aliasing, thereby resulting in an iid channel. This is confirmed by the plots in Fig. 13(e) and (f).

\section{CONCLUSIONS}

Fundamental understanding of the interaction between the signal space and the channel is key to reliable communication near capacity. The virtual representation framework introduced in this paper captures the essence of such interaction in the spatial dimension afforded by multiantenna systems. For uncorrelated scattering, we show that the channel matrix forms a segment of a stationary process and that the virtual channel matrix serves as its uncorrelated spectral representation. Via the notions of virtual path partitioning and spatial zooming/aliasing, the framework also provides a transparent characterization of the effects of physical scattering and array characteristics on channel statistics, capacity, and diversity. We have presented the essential ideas here, and more work needs to be done to fully develop the framework and to enable its practical application. We are currently working on augmenting the spatial framework in this paper to include temporal and spectral dimensions that leverages our recent work on time- and frequency-selective channels [19], [20].

One of the most promising potential applications of this work is the development of space-time coding techniques (see, e.g., [3]) for realistic channels. This is facilitated by the fact that $\boldsymbol{H}_{V}$ decomposes an arbitrary clustered channel into independent subchannels whose structure is very similar to the iid model exploited in existing space-time coding techniques; the sub-channels are represented by nonvanishing sub-matrices of $H_{V}$ with approximately uncorrelated entries. One direction for refining the ideas in this paper is the investigation of alternative spatial basis functions that could entail better smoothing properties and/or account for the effects of mutual antenna coupling as well as arbitrary array geometries. Prolate spheroidal and wavelet bases could be promising candidates in this context. Another direction is the development of models that are more accurate than the $k$-diagonal model to reflect the nature of scattering in each cluster. Finally, testing the ideas presented here in an experimental setup would be invaluable in refining the framework. Interestingly, certain types of antenna arrays, namely, lens arrays [21], inherently perform a spatial Fourier transform in the analog front-end. We are currently collaborating with researchers at the University of Colorado to further investigate this connection and its implications for practical design of multiantenna wireless communication systems.

\section{ACKNOWLEDGMENT}

The author gratefully acknowledges insightful discussions with H. Bolsckei, R. Heath, K. Liu, and V. Veeravalli, as well as the useful feedback provided by the reviewers.

\section{REFERENCES}

[1] G. J. Foschini, "Layered space-time architecture for wireless communication in a fading environment when using multi-element antennas," Bell Labs Tech. J., vol. 1, no. 2, pp. 41-59, 1996.

[2] E. Telatar, "Capacity of multi-antenna gaussian channels," AT\&T-Bell Labs Inter. Tech. Memo, 1995.

[3] V. Tarokh, N. Seshadri, and A. R. Calderbank, "Space-time codes for high data rate wireless communication: Performance criteria and code construction," IEEE Trans. Inform. Theory, vol. 44, pp. 744-765, Mar. 1998.

[4] A. J. Paulraj and C. B. Papadias, "Space-time processing for wireless communications," IEEE Signal Processing Mag., pp. 49-83, Nov. 1997.

[5] D. Gesbert, H. Bölcskei, D. A. Gore, and A. J. Paulraj, "Outdoor MIMO wireless channels: Models and performance prediction," IEEE Trans. Commun., July 2000, submitted for publication.

[6] D. Shiu, G. Foschini, M. Gans, and J. Kahn, "Fading correlation and its effect on the capacity of multielement antenna systems," IEEE Trans. Commun., vol. 48, pp. 502-513, Mar. 2000.

[7] G. G. Raleigh and J. M. Cioffi, "Spatio-temporal coding for wireless communication," IEEE Trans. Commun., vol. 46, pp. 357-366, Mar. 1998.

[8] D. H. Johnson and D. E. Dudgeon, Array Signal Processing. Englewood Cliffs, NJ: Prentice-Hall, 1993.

[9] J. Luo, J. R. Zeidler, and S. McLaughlin, "Performance analysis of compact antenna arrays with MRC in correlated Nakagami fading channels," IEEE Trans. Vehic. Tech., vol. 50, pp. 267-277, Jan. 2001.

[10] T. Svantesson and A. Ranheim, "Mutual coupling effects on the capacity of multielement antenna systems," in Proc. ICASSP, May 2001.

[11] W. C. Jakes, "Mobile radio propagation," in Microwave Mobile Communications, W. C. Jakes, Ed. New York: Wiley, 1974, pp. 11-78.

[12] D. Asztély, B. Ottersten, and A. L. Swindlehurst, "Generalized array manifold model for wireless communication channels with local scattering," Proc. Inst. Elect. Eng.,, Radar, Sonar Navig., vol. 145, pp. 51-57, Feb. 1998.

[13] J. Fuhl, A. F. Molisch, and E. Bonek, "Unified channel model for mobile radio systems with smart antennas," Proc. Inst. Elect. Eng., Radar, Sonar Navig., vol. 145, pp. 32-41, Feb. 1998. 
[14] P. A. Bello, "Characterization of randomly time-variant linear channels," IEEE Trans. Commun. Syst., vol. CS-11, pp. 360-393, Nov. 1963.

[15] H. Stark and J. W. Woods, Probability, Random Processes, and Estimation Theory for Engineers. Englewood Cliffs, NJ: Prentice-Hall, 1986.

[16] J. W. Brewer, "Kronecker products and matrix calculus in system theory," IEEE Trans. Circuits Syst., vol. CAS-25, pp. 772-781, Sept. 1978.

[17] A. Moustakas, H. Baranger, L. Balents, A. Sengupta, and S. Simon, "Communication through a diffusive medium: Coherence and capacity," Science, vol. 287 , p. 287,2000

[18] L. H. Ozarow, S. Shamai, and A. D. Wyner, "Information theoretic considerations for cellular mobile radio," IEEE Trans. Vehic. Tech., vol. 43, pp. 359-378, June 1994.

[19] A. M. Sayeed and B. Aazhang, "Joint multipath-Doppler diversity in mobile wireless communications," IEEE Trans. Commun., vol. 47, pp. 123-132, Jan. 1999.

[20] K. Liu, T. Kadous, and A. M. Sayeed, "Orthogonal time-frequency signaling for doubly dispersive channels," IEEE Trans. Inform. Theory, June 2001, submitted for publication.

[21] J. Vian and Z. Popović, "Smart lens antenna arrays," presented at the IEEE 2001 Int. Microwave Symp., Phoenix, AZ, June 2001.

[22] R. Heath, private communication

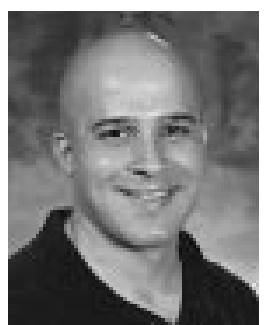

Akbar M. Sayeed (S'89-M'97-SM'02) received the B.S. degree from the University of Wisconsin-Madison in 1991 and the M.S. and Ph.D degrees in 1993 an 1996, respectively, from the University of Illinois at Urbana-Champaign, all in electrical and computer engineering.

While at the University of Illinois, he was a Research Assistant with the Coordinated Science Laboratory and was also the Schlumberger Fellow in signal processing from 1992 to 1995. From 1996 to 1997, he was a postdoctoral fellow at Rice University, Houston, TX. Since August 1997, he has been with the University of Wisconsin-Madison, where he is currently an Assistant Professor of electrical and computer engineering. His research interests are in wireless communications, sensor networks, statistical signal processing, wavelets, and time-frequency analyis.

Dr. Sayeed received the NSF CAREER Award in 1999 and the ONR Young Investigator Award in 2001. He served as an Associate Editor for the IEEE SigNAL PROCESSING LETTERS from 1999 to 2002. 